\title{
Learning by extrapolation from marginal to full-multivariate probability distributions: decreasingly naive Bayesian classification
}

\author{
Geoffrey I. Webb • Janice R. Boughton • Fei Zheng • \\ Kai Ming Ting • Houssam Salem
}

Received: 8 December 2009 / Accepted: 15 September 2011 / Published online: 13 October 2011

(C) The Author(s) 2011

\begin{abstract}
Averaged $n$-Dependence Estimators (A $n \mathrm{DE})$ is an approach to probabilistic classification learning that learns by extrapolation from marginal to full-multivariate probability distributions. It utilizes a single parameter that transforms the approach between a lowvariance high-bias learner (Naive Bayes) and a high-variance low-bias learner with Bayes optimal asymptotic error. It extends the underlying strategy of Averaged One-Dependence Estimators (AODE), which relaxes the Naive Bayes independence assumption while retaining many of Naive Bayes' desirable computational and theoretical properties. AnDE further relaxes the independence assumption by generalizing AODE to higher-levels of dependence. Extensive experimental evaluation shows that the bias-variance trade-off for Averaged 2-Dependence Estimators results in strong predictive accuracy over a wide range of data sets. It has training time linear with respect to the number of examples, learns in a single pass through the training data, supports incremental learning, handles directly missing values, and is robust in the face of noise. Beyond the practical utility of its lower-dimensional variants, $\mathrm{A} n \mathrm{DE}$ is of interest in that it demonstrates that it is possible to create low-bias high-variance generative learners and suggests strategies for developing even more powerful classifiers.
\end{abstract}

Keywords Bayesian learning · Classification learning · Probabilistic learning · Averaged one-dependence estimators · Naive Bayes · Semi-naive Bayesian learning · Learning without model selection $\cdot$ Ensemble learning $\cdot$ Feating

Editor: Peter Flach.

G.I. Webb $(\bowtie) \cdot$ J.R. Boughton · F. Zheng · K.M. Ting · H. Salem

Faculty of Information Technology, Monash University, Clayton, VIC, 3800, Australia

e-mail: Geoff.Webb@monash.edu

J.R. Boughton

e-mail: Janice.Boughton@monash.edu

K.M. Ting

e-mail: Kaiming.Ting@monash.edu 


\section{Introduction}

This paper presents a family of learning algorithms that utilize a predefined function to extrapolate from observed marginal distributions to the full multivariate distribution of interest. This stands in contrast to the majority of learning algorithms that instead seek to fit a model directly to the observed multivariate probability distribution. Whereas learning is sometimes cast as a problem of searching through a space of hypotheses to find one that best fits the training data (Mitchell 1982), this new approach does not employ search and does not perform model selection.

The members of this new family of algorithms have a unique combination of features that is well suited to many applications. We discuss these features in more detail below. Notable amongst them are training complexity linear with respect to the number of training examples; single pass learning; direct capacity for incremental learning; and accuracy that is competitive with the state-of-the-art. They are of further theoretical interest because they demonstrate that it is possible to create low bias generative learners.

The family contains algorithms that range from low variance coupled with high bias through to high variance coupled with low bias. Successive members of the family will be best suited to differing quantities of data, starting with low variance for small data, with successively lower bias but higher variance suiting ever increasing data quantities (Brain and Webb 2002). The asymptotic error of the lowest bias variant is Bayes optimal.

One member of this family of algorithms, naive Bayes (NB), is already well known. A second member, Averaged One-Dependence Estimators (AODE) (Webb et al. 2005), has enjoyed considerable popularity since its introduction in 2005 (Nikora 2005; Camporelli 2006; Flikka et al. 2006; Orhan and Altan 2006; Lasko et al. 2006; Hunt 2006; Ferrari and Aitken 2006; Birzele and Kramer 2006; Kunchevaa et al. 2007; Lau et al. 2007; Masegosa et al. 2007; Wang et al. 2007; Garcia et al. 2008; Tian et al. 2008; Kurz et al. 2009; Leon et al. 2009; Shahri and Jamil 2009; Simpson et al. 2009; Affendey et al. 2010; García-Jiménez et al. 2010; Hopfgartner et al. 2010; Liew et al. 2010). The work presented in this paper arises from the realization that NB and AODE are but two instances of a family of algorithms, which we call $\mathrm{A} n \mathrm{DE}$.

In Sect. 2 we explain the underlying learning strategy, and define the AnDE family of algorithms. The A $n \mathrm{DE}$ family of algorithms build upon the method pioneered by AODE (Webb et al. 2005). In Sect. 3 we discuss how the AnDE algorithms relate to Feating (Ting et al. 2011), a generic approach to ensembling that also builds upon techniques pioneered by AODE. In Sect. 4 we present an extensive evaluation of the AnDE family of algorithms, comparing their performance to relevant Bayesian techniques, to Feating and to the state-ofthe-art Random Forests classifier. Section 5 presents conclusions and directions for future research.

\section{The AnDE family of algorithms}

We wish to estimate from a training sample $\mathcal{T}$ of $t$ classified objects the probability $\mathrm{P}(y \mid \mathbf{x})$ that an example $\mathbf{x}=\left\langle x_{1}, \ldots, x_{a}\right\rangle$ belongs to class $y$, where $x_{i}$ is the value of the $i$ th attribute and $y \in\left\{c_{1}, \ldots, c_{k}\right\}$. We use $\bar{v}$ to denote the average number of values per attribute. These and other elements of notation are listed in Table 1.

From the definition of conditional probability we have

$$
\mathrm{P}(y \mid \mathbf{x})=\mathbf{P}(y, \mathbf{x}) / \mathrm{P}(\mathbf{x})
$$


Table 1 Notation

\begin{tabular}{ll}
\hline $\mathrm{P}(e)$ & The unconditioned probability of event $e$ \\
$\mathrm{P}(e \mid w)$ & The conditional probability of event $e$ given event $w$ \\
$\hat{\mathrm{P}}(e)$ & An estimate of $\mathrm{P}(e)$ \\
$\hat{\mathrm{P}}_{\mathrm{NB}}(e)$ & A naive Bayes estimate of $\mathrm{P}(e)$ \\
$\hat{\mathrm{P}}_{\mathrm{AODE}}(e)$ & An AODE estimate of $\mathrm{P}(e)$ \\
$\hat{\mathrm{P}}_{\mathrm{A} n \mathrm{DE}}(e)$ & An A $n$ DE estimate of $\mathrm{P}(e)$ \\
$a$ & The number of attributes \\
$c_{i}$ & The $i$ th class \\
$k$ & The number of classes \\
$t$ & The number of training examples in $\mathcal{T}$ \\
$\bar{v}$ & The average number of values per attribute \\
$y$ & A value from the set of all classes $\left\{c_{1}, \ldots, c_{k}\right\}$ \\
$\mathcal{T}$ & A training sample of $t$ classified objects \\
$\mathbf{x}=\left\langle x_{1}, \ldots, x_{a}\right\rangle$ & An object \\
$x_{i}$ & The value of the $i$ th attribute of $\mathbf{x}=\left\langle x_{1}, \ldots, x_{a}\right\rangle$ \\
$x_{\{i, j, \ldots, q\}}$ & The subset of attributes values from $\mathbf{x}$ with the specified indices. For example, \\
$\left(\begin{array}{l}\mathcal{A} \\
n\end{array}\right)$ & $x_{\{2,3,5\}=\left\langle x_{2}, x_{3}, x_{5}\right\rangle}$ \\
$\delta\left(x_{\alpha}\right)$ & The set of all size- $n$ subsets of $\{1, \ldots, a\}$ \\
\hline & A function that is 1 if $\mathcal{T}$ contains an object with the value $x_{\alpha}$, otherwise 0
\end{tabular}

As $\mathrm{P}(\mathbf{x})=\sum_{i=1}^{k} \mathrm{P}\left(c_{i}, \mathbf{x}\right)$, we can always estimate (1) from estimates of $\mathrm{P}(y, \mathbf{x})$ for each class using

$$
\mathrm{P}(y, \mathbf{x}) / \mathrm{P}(\mathbf{x})=\mathrm{P}(y, \mathbf{x}) / \sum_{i=1}^{k} \mathrm{P}\left(c_{i}, \mathbf{x}\right) .
$$

In consequence, in the remainder of this paper we consider only the problem of estimating $\mathrm{P}(y, \mathbf{x})$, thereby setting the work in a generative framework.

We define the dimensionality of a probability or probability estimate as the number of attributes in the distribution to which the probability or estimate relates. Hence, the dimensionality of $\mathrm{P}(y, \mathbf{x})$ is $a+1$.

If the training data do not include sufficient examples of $\mathbf{x}$ to directly derive accurate estimates of each $\mathrm{P}\left(c_{i}, \mathbf{x}\right)$, we must extrapolate these estimates from observations of lowerdimensional probabilities in the data. All other things being equal, an estimate of a lowerdimensional probability from a given finite training set will be more accurate than an estimate of a higher-dimensional probability, and estimates of higher-dimensional probabilities will vary more from training sample to training sample. Hence, models derived from lowerdimensional probability estimates are likely to have lower variance than models derived from higher-dimensional probability estimates. On the other hand, models derived from higher-dimensional probabilities are likely to have lower bias, as less restrictive assumptions are made about the form of the probability distribution.

This is illustrated in Fig. 1, that shows a simple attribute-space with three ternary attributes and a binary class. To classify a new object with attribute-values Age = Old, Pulse $=$ Slow and Temperature $=$ High, one wishes to infer the class distribution in the cell highlighted in Fig. 1(a), which is a four-dimensional probability distribution. If there are insufficient examples to directly estimate that distribution, it might be extrapolated 


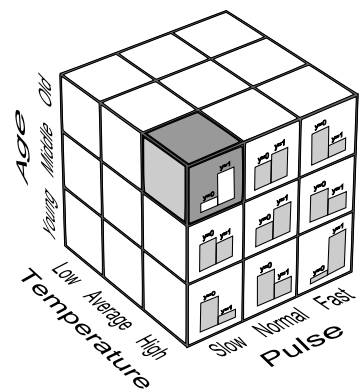

$\mathrm{P}(y \wedge$ Age $=$ Old $\wedge$ Pulse $=$ Slow $\wedge$ Temperature $=$ High)

(a)

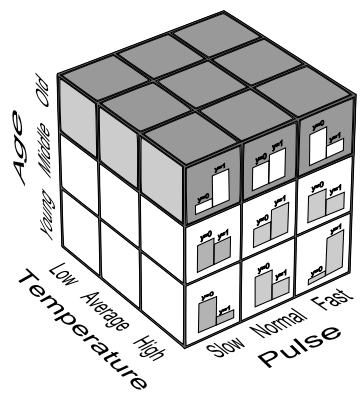

$\mathrm{P}(y \wedge$ Age $=$ Old $)$

(c)

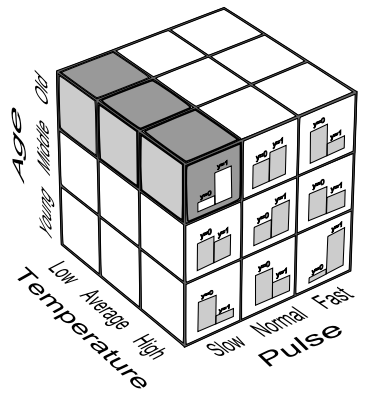

$$
\begin{array}{r}
\mathrm{P}(y \wedge \text { Age }=\text { Old } \wedge \\
\text { Pulse }=\text { Slow })
\end{array}
$$

(f)

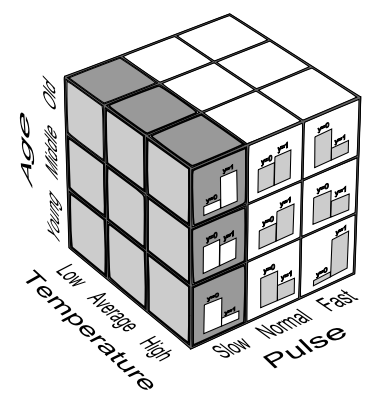

$\mathrm{P}(y \wedge$ Pulse $=$ Slow $)$

(d)

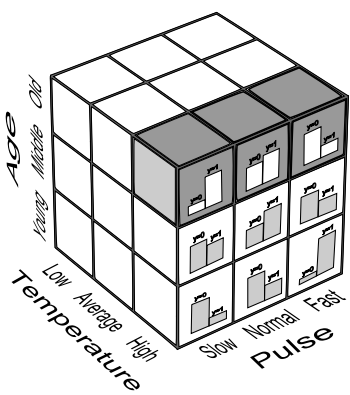

$$
\mathrm{P}(y \wedge \text { Age }=\text { Old } \wedge
$$

Temperature $=$ High)

(g)

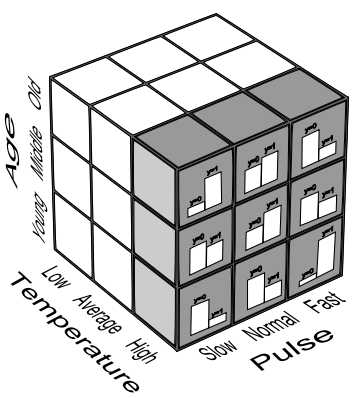

$\mathrm{P}(y \wedge$ Temperature $=$ High $)$

(e)

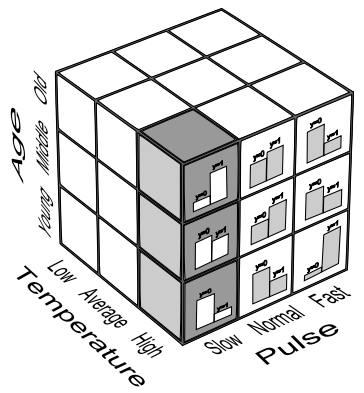

$\mathrm{P}(y \wedge$ Pulse $=$ Slow $\wedge$

Temperature $=$ High)

(h)

Fig. 1 Probabilities of varying dimensionality for an attribute-space with three ternary attributes and a binary class

from any of a number of lower dimensional probability distributions. The prior class distribution $\mathrm{P}(y)$ is a one-dimensional probability distribution that can be estimated from the entire attribute-space (Fig. 1(b)). The two-dimensional probabilities $\mathrm{P}(y \wedge A g e=$ Old $)$, $\mathrm{P}(y \wedge$ Pulse $=$ Slow $), \mathrm{P}(y \wedge$ Temperature $=$ High $)$ can be estimated from the regions depicted in Fig. 1(c-e). The regions associated with the three-dimensional probabili- 
ties $\mathrm{P}(y \wedge$ Age $=$ Old $\wedge$ Pulse $=$ Slow $), \mathrm{P}(y \wedge$ Age $=$ Old $\wedge$ Temperature $=$ High $)$ and $\mathrm{P}(y \wedge$ Pulse $=$ Slow $\wedge$ Temperature $=$ High $)$ are illustrated in Fig. 1(f-h).

From the definition of conditional probability we have

$$
\mathrm{P}(y, \mathbf{x})=\mathrm{P}(y) \mathrm{P}(\mathbf{x} \mid y) \text {. }
$$

If the number of classes, $k$, is small, it should be possible to obtain a sufficiently accurate estimate of $\mathrm{P}(y)$ from the sample frequencies. However, we still have the problem that $\mathbf{x}$ may not occur sufficiently frequently in the training data and hence accurate estimates of $\mathrm{P}(\mathbf{x} \mid y)$ cannot be obtained directly from the sample frequencies.

The solution used by NB is to extrapolate to $\hat{\mathrm{P}}(\mathbf{x} \mid y)$ from each two-dimensional probability estimate $\hat{\mathrm{P}}\left(x_{i} \mid y\right)$ by assuming the attributes are independent given the class:

$$
\mathrm{P}(\mathbf{x} \mid y)=\prod_{i=1}^{a} \mathrm{P}\left(x_{i} \mid y\right) .
$$

Hence we classify using

$$
\hat{\mathrm{P}}_{\mathrm{NB}}(y, \mathbf{x})=\hat{\mathrm{P}}(y) \prod_{i=1}^{a} \hat{\mathrm{P}}\left(x_{i} \mid y\right) .
$$

With reference to Fig. 1, NB estimates the distribution in (a) by extrapolation from the distributions in (b) (that gives $\hat{\mathrm{P}}(y)$ ), (c) (that gives $\hat{\mathrm{P}}($ Age $=$ old $\mid y)$ ), (d) (that gives $\hat{\mathrm{P}}($ Pulse $=$ slow $\mid y))$ and (e) (that gives $\hat{\mathrm{P}}($ Temperature $=$ high $\mid y))$.

The independence assumption is a very strong assumption about the underlying probability distribution. As a result, NB has very high bias. However, due to the low dimensionality of the base probabilities from which the model is estimated, it has low variance.

\subsection{AODE}

Averaged One-Dependence Estimators (AODE) (Webb et al. 2005) extends to threedimensional probabilities NB's search-free strategy of extrapolation from lower-dimensional probabilities. It does so by averaging the estimates of all of a class of three-dimensional estimators.

A Super-Parent One-Dependence Estimator (SPODE) is a three-dimensional probability estimator that relaxes the assumption of conditional independence by making all other attributes independent given the class and one privileged attribute, the super-parent, $x_{\alpha}$. This is a weaker conditional independence assumption than NB's, as it is necessarily true if NB's is true and may also be true when NB's is not.

It uses

$$
\mathrm{P}(y, \mathbf{x})=\mathrm{P}\left(y, x_{\alpha}\right) \mathrm{P}\left(\mathbf{x} \mid y, x_{\alpha}\right)
$$

together with a conditional independence assumption

$$
\mathrm{P}\left(\mathbf{x} \mid y, x_{\alpha}\right)=\prod_{i=1}^{a} \mathrm{P}\left(x_{i} \mid y, x_{\alpha}\right) .
$$

As this is a weaker assumption than (4), the bias of the model should be lower than that of NB. However, it is derived from higher-dimensional probability estimates and hence its variance should be higher. 
AODE exploits the lower bias of SPODEs while addressing their higher variance by averaging over all estimates of $\mathrm{P}(y, \mathbf{x})$ produced by using different super-parents. AODE seeks to use

$$
\hat{\mathrm{P}}(y, \mathbf{x})=\sum_{\alpha=1}^{a} \hat{\mathrm{P}}\left(y, x_{\alpha}\right) \hat{\mathrm{P}}\left(\mathbf{x} \mid y, x_{\alpha}\right) / a .
$$

However, in practice it is desirable to only use estimates of probabilities for which relevant examples occur in the data. Hence, AODE actually uses

$$
\hat{\mathrm{P}}_{\mathrm{AODE}}(y, \mathbf{x})= \begin{cases}\sum_{\alpha=1}^{a} \delta\left(x_{\alpha}\right) \hat{\mathrm{P}}\left(y, x_{\alpha}\right) \hat{\mathrm{P}}\left(\mathbf{x} \mid y, x_{\alpha}\right) / \sum_{\alpha=1}^{a} \delta\left(x_{\alpha}\right) & : \sum_{\alpha=1}^{a} \delta\left(x_{\alpha}\right)>0 \\ \hat{\mathrm{P}}_{\mathrm{NB}}(y, \mathbf{x}) & : \text { otherwise }\end{cases}
$$

where $\delta\left(x_{\alpha}\right)$ is 1 if attribute-value $x_{\alpha}$ is present in the data, otherwise 0 . That is, it averages over all superparents whose value occurs in the data, and defaults to NB if there are no such superparents.

As AODE uses all of a predefined family of estimators, each of which extrapolates the desired high-dimensional probability from lower-dimensional probabilities, it does not perform search.

In terms of the example attribute space, AODE extrapolates to Fig. 1(a) from the lowerdimensional probabilities illustrated in Fig. 1(c-h) with (f) conditioned on (c) and (d), (g) conditioned on (c) and (e), and (g) conditioned on (d) and (e).

AODE has demonstrated strong prediction accuracy (both zero-one loss and meansquared error) with relatively modest computational requirements for low dimensional data (Webb et al. 2005). In consequence, it has enjoyed substantial uptake (Nikora 2005; Camporelli 2006; Flikka et al. 2006; Orhan and Altan 2006; Lasko et al. 2006; Hunt 2006; Ferrari and Aitken 2006; Birzele and Kramer 2006; Kunchevaa et al. 2007; Lau et al. 2007; Masegosa et al. 2007; Wang et al. 2007; Garcia et al. 2008; Tian et al. 2008; Kurz et al. 2009; Leon et al. 2009; Shahri and Jamil 2009; Simpson et al. 2009; Affendey et al. 2010; García-Jiménez et al. 2010; Hopfgartner et al. 2010; Liew et al. 2010).

\section{$2.2 \mathrm{~A} n \mathrm{DE}$}

In this paper we generalize to higher-dimensional probabilities the strategy of search-free extrapolation from lower-dimensional probabilities.

For notational convenience we define

$$
x_{\{i, j, \ldots, q\}}=\left\langle x_{i}, x_{j}, \ldots, x_{q}\right\rangle .
$$

For example, $x_{\{2,3,5\}}=\left\langle x_{2}, x_{3}, x_{5}\right\rangle$.

$\mathrm{A} n \mathrm{DE}$ aims to use

$$
\hat{\mathrm{P}}(y, \mathbf{x})=\sum_{s \in\left(\begin{array}{c}
\mathcal{A} \\
n
\end{array}\right)} \hat{\mathrm{P}}\left(y, x_{s}\right) \hat{\mathrm{P}}\left(\mathbf{x} \mid y, x_{s}\right) /\left(\begin{array}{l}
a \\
n
\end{array}\right),
$$

where $\left(\begin{array}{c}\mathcal{A} \\ n\end{array}\right)$ indicates the set of all size- $n$ subsets of $\{1, \ldots, a\}$. 
However, in practice we also need to avoid using pairs of superparents whose values do not occur in the data, and hence use

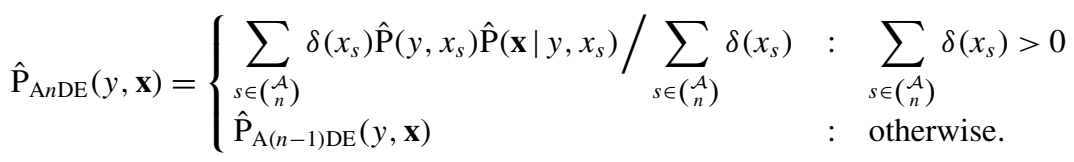

Attributes are assumed independent given the superparents and the class. Hence, $\mathrm{P}\left(\mathbf{x} \mid y, x_{s}\right)$ is estimated by

$$
\hat{\mathrm{P}}\left(\mathbf{x} \mid y, x_{s}\right)=\prod_{i=1}^{a} \hat{\mathrm{P}}\left(x_{i} \mid y, x_{s}\right) .
$$

Note that $\mathrm{P}\left(x_{i} \mid y, x_{s}\right)=1$ when $i \in s$. Whereas other probability estimates should be smoothed or regularized, smoothed estimates should not be used in this case, and in practice these values are not included in the calculation.

It should be recalled that AODE is NB and A1DE is AODE.

In terms of the simple attribute-space depicted in Fig. 1, A2DE extrapolates to (a) from (a) conditioned on each of (f), (g) and (h), and A3DE makes inferences directly from the class distribution in (a).

When $n=a,\left(\begin{array}{c}\mathcal{A} \\ n\end{array}\right)=\{\{1, \ldots a\}\}$, so $x_{s}=\mathbf{x}$. Therefore, the ultimate expression of A $n \mathrm{DE}$, $\mathrm{A} a \mathrm{DE}$ seeks to classify using

$$
\hat{\mathrm{P}}_{\mathrm{A} a \mathrm{DE}}(y, \mathbf{x})=\hat{\mathrm{P}}(y, \mathbf{x}) \hat{\mathrm{P}}(\mathbf{x} \mid y, \mathbf{x}) /\left(\begin{array}{l}
a \\
a
\end{array}\right)
$$

where $\hat{\mathrm{P}}(y, \mathbf{x})$ is estimated directly from $\mathcal{T}$, cascading to ever lower dependence estimators should the combination of attribute-values not be present in $\mathcal{T}$. As $\mathrm{P}(\mathbf{x} \mid y, \mathbf{x})$ and $\left(\begin{array}{l}a \\ a\end{array}\right)$ both equal 1, it classifies using only its direct estimate of $\mathrm{P}(y, \mathbf{x})$.

Observation 1 The asymptotic classification performance of $\mathrm{A} a \mathrm{DE}$ equals that of the Bayes optimal classifier.

Proof A $a \mathrm{DE}$ classifies using

$$
\underset{y}{\operatorname{argmax}}\left(\hat{\mathrm{P}}(y, \mathbf{x}) / \sum_{z \in\left\{c_{1}, \ldots, c_{k}\right\}} \hat{\mathrm{P}}(z, \mathbf{x})\right)
$$

where each $\hat{\mathrm{P}}(\cdot)$ is directly estimated from the observed data and hence approaches $\mathrm{P}(\cdot)$ as the quantity of data approaches infinity. Hence, in the limit, $\mathrm{A} a \mathrm{DE}$ approaches

$$
\underset{y}{\operatorname{argmax}}\left(\mathrm{P}(y, \mathbf{x}) / \sum_{z \in\left\{c_{1}, \ldots, c_{k}\right\}} \mathrm{P}(z, \mathbf{x})\right)
$$

which is the Bayes optimal classifier.

However, assuming there is sufficient data to compute the necessary probabilities, and we wish to store the necessary probabilities rather than computing them as required for 
classification, the space complexity of $\mathrm{A} a \mathrm{DE}$ is $O\left(k \bar{v}^{a}\right)$. This is because joint frequencies must be stored for every combination of attribute value and class value. Except in cases of low dimensional data, even the computational requirements of $\mathrm{A} 3 \mathrm{DE}$ defeat our Weka implementation, and hence in this paper we present primarily results for A2DE with some illustrative examples of A3DE.

$\mathrm{A} n \mathrm{DE}$ forms an $(n+2)$-dimensional probability table containing the observed frequency for each combination of $n+1$ attribute values and the class labels. The space complexity of the table is $O\left(k\left(\begin{array}{c}a \\ n+1\end{array}\right) \bar{v}^{n+1}\right)$ and the time complexity of compiling it is $O\left(t\left(\begin{array}{c}a \\ n+1\end{array}\right)\right)$, as we need to update each entry for every combination of the $n+1$ attribute-values for every instance. The time complexity of classifying a single example is $O\left(k a\left(\begin{array}{l}a \\ n\end{array}\right)\right)$ as we need to consider each attribute for every qualified combination of $n$ parent attributes within each class.

We demonstrate that as $n$ increases, averaged $n$-dependence estimators achieve lower bias at the cost of higher variance. In consequence, the ideal dimensionality of dependence will depend on the degree to which the underlying probability distribution fits the assumptions of the $n$-dependence estimator, the quantity of data available to estimate the base probabilities, and the computational demands of averaging over higher-dimensional estimators.

\subsection{Weighted averaging}

AODE and its generalization AnDE perform an unweighted average of the component $n$ dependence estimators. It has been demonstrated that weighted averaging can improve upon the accuracy of AODE's estimates (Cerquides and Mántaras 2005; Jiang and Zhang 2006; Yang et al. 2007). The empirical evidence suggests that the Bayesian model averaging of Maximum a Posteriori Linear Mixture of Generative Distributions (MAPLMG) is the most effective of current approaches (Cerquides and Mántaras 2005; Yang et al. 2007). It seems likely that similar approaches will be equally effective with $n$-dependence estimators.

It is notable that the introduction of Bayesian model averaging to the $\mathrm{A} n \mathrm{DE}$ framework introduces both search and discriminative learning, as a search is performed for the set of weights that optimize the posterior probabilities relative to the training data. Doing so can be expected to reduce bias at the cost of introduction of variance.

One of the interesting questions that this paper investigates is the relative payoff for the investment of additional computation in either performing Bayesian model averaging on $\mathrm{A} n \mathrm{DE}$ or increasing $n$ and using $\mathrm{A}(n+1) \mathrm{DE}$. Both approaches can be expected to reduce bias at the cost of an increase in both variance and computation. Which provides the more effective trade-off?

\subsection{Tree Augmented Naive Bayes}

An $n$-dependence Bayesian classifier ( $n$-DBC) (Sahami 1996) is a Bayesian network in which each attribute depends upon the class and at most $n$ other non-class attributes. An $n$ DBC uses $(n+2)$-dimensional probabilities. Within this framework, NB is a 0 -DBC, AODE is a $1-\mathrm{DBC}$ and the full Bayesian classifier is an $(a+1)$-DBC.

An alternative to the $\mathrm{A} n \mathrm{DE}$ approach to relaxing NB's independence assumption is to use search to select a single model that adds selected interdependencies between attributes. Tree Augmented Naive Bayes (TAN) (Friedman et al. 1997) is a popular approach of this type. It uses conditional mutual information to select a best single parent for each attributes, in addition to the class. Thus, it is a 1-DBC. 
It is interesting to consider how search for a single Bayesian classifier model compares with averaging over a class of Bayesian classifier models of the same level of dependence or of a higher level of dependence. This paper also investigates this issue.

\section{Relationship to Feating}

Feating (Ting et al. 2011) is a generic ensemble learning technique that also builds upon the ensembling strategy of AODE. Like A $n$ DE, Feating operates by building a local model for each combination of $n$ attribute values. To classify a new instance, Feating applies all applicable local models and aggregates the results by performing a majority vote of the resulting classifications. AnDE is similar to Feating NB. However, Feating aggregates the predictions of its base learners by taking the mode of the class predictions. For probabilistic classifiers, these class predictions correspond to the maximum posterior probability. In contrast, $\mathrm{A} n \mathrm{DE}$ uses the ensemble to estimate the joint probability, $\mathrm{P}(\mathbf{x}, y)$ for each class, and then calculates its estimate of the posterior probability from this ensembled estimate of the joint probability. A generic ensembling technique, such as Feating, cannot work by calculating an ensemble estimate of the joint probabilities because many classifiers do not produce appropriate probability estimates.

Despite the close relationship to Feating, $\mathrm{A} n \mathrm{DE}$ is worthy of study in its own right for three reasons.

First, irrespective of which aggregation method is used, coupling the search-less ensembling strategy embodied by Feating with search-less base learner NB creates a learner that can deliver low bias using a predefined mapping from low dimensional probabilities to the desired high dimensional probabilities without search or model selection. Hence, AnDE provides an example of an alternative to the traditional search-based learning paradigm which is able to deliver low bias classifiers.

Second, as already noted, A $n$ DE utilizes a different aggregation method to Feating. It is interesting to examine the consequences of these differences. Cerquides and de Màntaras (2005) found that weighted ensembles of joint probability estimates achieved lower error than weighted ensembles of posterior probability estimates, so there is some evidence that the outcomes may be substantially different.

Third, as there is overlap in the information required by each of its local models, A $n$ DE can use a single compiled matrix of joint frequencies, saving considerable space relative to storing all of the local models independently. The space complexity of an A $n$ DE model is $O\left(k\left(\begin{array}{c}a \\ n+1\end{array}\right)\right) \bar{v}^{n+1}$ whereas the space complexity of Feating NB to level $n$ is $O\left(k(a-n)\left(\begin{array}{l}a \\ n\end{array}\right) \bar{v}^{n+1}\right)$, which is $(n+1)$ times the space complexity of AnDE. Most base models formed by Feating will not have this property, and hence AnDE is a notable special case.

\section{Evaluation}

In this section, we evaluate the efficacy of $\mathrm{A} n \mathrm{DE}$. Due to relatively high time complexity of higher-dimensional estimators, the highest level of AnDE with which we perform detailed assessment is A2DE. The primary metrics we use are bias, variance, zero-one loss and RMSE. To assess computational overheads we use total training and classification times divided by the number of examples. 
We first study the performance of NB, AODE and A2DE to reveal how performance varies as $n$ increases within the AnDE framework. TAN and MAPLMG are studied to show how the search-free generative $\mathrm{A} n \mathrm{DE}$ strategy compares with, respectively, discriminative search for a single Bayesian network classifier of the same dimensionality of dependence, and discriminative search for a weighted classifier of the next lower dimensionality of dependence. We also compare A2DE, that estimates the mean joint probability of the submodels, with variants that calculate the mean posterior probability (PA2DE) and perform Feating of NB by taking the mode of the class predictions of the submodels (FA2DE). Finally, to explore how the classification performance of A2DE compares to state-of-the-art classifiers, we also study Random Forests (Breiman 2001) with ten trees (RF10) and Random Forests with 100 trees (RF100).

We compare these algorithms implemented in the Weka workbench (Witten and Frank 2005) on the 62 data sets described in Table 2 that have been used previously in related

Table 2 Data sets used for experiments

\begin{tabular}{|c|c|c|c|c|}
\hline No. & Domain & Case & Att & Class \\
\hline 1 & Abalone & 4177 & 9 & 3 \\
\hline 2 & Adult & 48842 & 15 & 2 \\
\hline 3 & Annealing & 898 & 39 & 6 \\
\hline 4 & Audiology & 226 & 70 & 24 \\
\hline 5 & Auto Imports & 205 & 26 & 7 \\
\hline 6 & Balance Scale & 625 & 5 & 3 \\
\hline 7 & Breast Cancer (Wisconsin) & 699 & 10 & 2 \\
\hline 8 & Car Evaluation & 1728 & 8 & 4 \\
\hline 9 & Census-Income (KDD) & 299285 & 40 & 2 \\
\hline 10 & Connect-4 Opening & 67557 & 43 & 3 \\
\hline 11 & Contact-lenses & 24 & 5 & 3 \\
\hline 12 & Contraceptive Method Choice & 1473 & 10 & 3 \\
\hline 13 & Covertype & 581012 & 55 & 7 \\
\hline 14 & Credit Screening & 690 & 16 & 2 \\
\hline 15 & Cylinder Bands & 540 & 40 & 2 \\
\hline 16 & Dermatology & 366 & 35 & 6 \\
\hline 17 & Echocardiogram & 131 & 7 & 2 \\
\hline 18 & German & 1000 & 21 & 2 \\
\hline 19 & Glass Identification & 214 & 10 & 3 \\
\hline 20 & Haberman's Survival & 306 & 4 & 2 \\
\hline 21 & Heart Disease (Cleveland) & 303 & 14 & 2 \\
\hline 22 & Hepatitis & 155 & 20 & 2 \\
\hline 23 & Horse Colic & 368 & 22 & 2 \\
\hline 24 & House Votes 84 & 435 & 17 & 2 \\
\hline 25 & Hungarian & 294 & 14 & 2 \\
\hline 26 & Hypothyroid(Garavan) & 3772 & 30 & 4 \\
\hline 27 & Ionosphere & 351 & 35 & 2 \\
\hline 28 & Iris Classification & 150 & 5 & 3 \\
\hline 29 & King-rook-vs-king-pawn & 3196 & 37 & 2 \\
\hline
\end{tabular}


Table 2 (Continued)

\begin{tabular}{|c|c|c|c|c|}
\hline No. & Domain & Case & Att & Class \\
\hline 30 & Labor negotiations & 57 & 17 & 2 \\
\hline 31 & LED & 1000 & 7 & 10 \\
\hline 32 & Letter Recognition & 20000 & 17 & 26 \\
\hline 33 & Liver Disorders (Bupa) & 345 & 7 & 2 \\
\hline 34 & Lung Cancer & 32 & 57 & 3 \\
\hline 35 & Lymphography & 148 & 19 & 4 \\
\hline 36 & MAGIC Gamma Telescope & 19020 & 11 & 2 \\
\hline 37 & Mushrooms & 8124 & 23 & 2 \\
\hline 38 & Nettalk (Phoneme) & 5438 & 8 & 52 \\
\hline 39 & New-Thyroid & 215 & 6 & 3 \\
\hline 40 & Nursery & 12960 & 9 & 5 \\
\hline 41 & Optical Digits & 5620 & 49 & 10 \\
\hline 42 & Page Blocks & 5473 & 11 & 5 \\
\hline 43 & Pen Digits & 10992 & 17 & 10 \\
\hline 44 & Pima Indians Diabetes & 768 & 9 & 2 \\
\hline 45 & Postoperative Patient & 90 & 9 & 3 \\
\hline 46 & Primary Tumor & 339 & 18 & 22 \\
\hline 47 & Promoter Gene Sequences & 106 & 58 & 2 \\
\hline 48 & Segment & 2310 & 20 & 7 \\
\hline 49 & Sick-euthyroid & 3772 & 30 & 2 \\
\hline 50 & Sign & 12546 & 9 & 3 \\
\hline 51 & Sonar Classification & 208 & 61 & 2 \\
\hline 52 & SPAM E-mail & 4601 & 58 & 2 \\
\hline 53 & Splice-junction Gene Sequences & 3190 & 62 & 3 \\
\hline 54 & Syncon & 600 & 61 & 6 \\
\hline 55 & Teaching Assistant Evaluation & 151 & 6 & 3 \\
\hline 56 & Tic-Tac-Toe Endgame & 958 & 10 & 2 \\
\hline 57 & Vehicle & 846 & 19 & 4 \\
\hline 58 & Volcanoes & 1520 & 4 & 4 \\
\hline 59 & Vowel & 990 & 14 & 11 \\
\hline 60 & Waveform-5000 & 5000 & 41 & 3 \\
\hline 61 & Wine Recognition & 178 & 14 & 3 \\
\hline 62 & Zoo & 101 & 17 & 7 \\
\hline
\end{tabular}

research (Webb et al. 2005; Langley and Sage 1994; Pazzani 1996; Domingos and Pazzani 1996; Zheng and Webb 2000; Yang et al. 2006). Each algorithm is tested on each data set using the repeated cross-validation bias-variance estimation method (Webb 2000). In order to maximize the variation in the training data from trial to trial, we use two-fold cross validation. To minimize the variance in our measurements we report average values over 50 cross-validation trials.

We also form learning curves for NB, AODE, A2DE and A3DE on the Adult data set to further investigate how increasing $n$ within the $\mathrm{A} n \mathrm{DE}$ framework affects performance as the quantity of data increases. 
The current implementations of AODE and A2DE are limited to categorical data. A number of approaches have been developed for extending AODE to numeric data (Flores et al. 2009). These could be generalized to the $\mathrm{A} n \mathrm{DE}$ framework, but how best to do so is a matter for future research. Hence, we assess only the relative capacities of these algorithms with respect to categorical data. To this end, all numeric attributes are discretized. When MDL discretization (Fayyad and Irani 1993), a common discretization method for NB, is used to discretize quantitative attributes within each cross-validation fold, many attributes have only one value. In these experiments, we discretize quantitative attributes using three-bin equal-frequency discretization prior to classification.

The base probabilities are estimated using $m$-estimation (Cestnik 1990) $(m=1)$, as it often appears to lead to more accurate probabilities than Laplace estimation for NB and AODE. An exception is that we always use 1.0 for $\hat{\mathrm{P}}\left(x_{i} \mid y, x_{s}\right)$ when $i \in s$.

The above experiments were conducted on a single CPU single core virtual Linux machine running on a Dell PowerEdge 1950 with dual quad core Intel Xeon E5410 processors running at $2333 \mathrm{MHz}$ with $32 \mathrm{~GB}$ of RAM. ${ }^{1}$

Average values for each combination of metric, algorithm and dataset are provided in the Appendix. Summary results are provided in the text.

\subsection{Varying $n$ within $\mathrm{A} n \mathrm{DE}$}

We first consider the relative performance of the three variants of $\mathrm{A} n \mathrm{DE}$. For each performance measure, the number of data sets for which A2DE has lower, equal or higher outcomes relative to AODE and NB are summarized into win/draw/loss records, and likewise for AODE relative to NB. For each win/draw/loss record a binomial sign test is performed to assess the probability of observing the given number of wins and losses if each were equally likely. These results are presented in Table 3 . As expected, we see that increasing $n$ from 0 (NB) to 1 (AODE) to 2 (A2DE) consistently decreases bias at the cost of an increase in variance. As we believe that different bias and variance profiles suit different data quantities (Brain and Webb 2002), we believe that the zero-one loss and RMSE results tell us as much about the composition of the data collection as they do about the algorithms. Specifically, we contend that whether one algorithm or another will win on a given dataset is determined

Table 3 Win/draw/loss: AnDE, $n=0,1$ and 2, on all 62 data sets

\begin{tabular}{|c|c|c|c|c|c|c|}
\hline & \multicolumn{2}{|c|}{ A2DE vs AODE } & \multicolumn{2}{|c|}{$\mathrm{A} 2 \mathrm{DE}$ vs NB } & \multicolumn{2}{|c|}{ AODE vs NB } \\
\hline & $\mathrm{W} / \mathrm{D} / \mathrm{L}$ & $p$ & $\mathrm{~W} / \mathrm{D} / \mathrm{L}$ & $p$ & $\mathrm{~W} / \mathrm{D} / \mathrm{L}$ & $p$ \\
\hline Bias & $47 / 0 / 15$ & $<0.001$ & $49 / 2 / 11$ & $<0.001$ & $48 / 0 / 14$ & $<0.001$ \\
\hline Variance & $19 / 1 / 42$ & $<0.001$ & $15 / 0 / 47$ & $<0.001$ & $20 / 1 / 41$ & 0.005 \\
\hline Zero-one loss & $33 / 2 / 27$ & 0.259 & $42 / 1 / 19$ & 0.002 & $44 / 1 / 17$ & $<0.001$ \\
\hline RMSE & $35 / 1 / 26$ & 0.153 & $15 / 0 / 47$ & $<0.001$ & $49 / 1 / 12$ & $<0.001$ \\
\hline
\end{tabular}

\footnotetext{
${ }^{1}$ Due to technical issues including memory leaks in the Weka implementation of Random Forests, it was not possible to complete all 50 runs of 2-fold cross validation for RF10 on Covertype and RF100 on Covertype and Census-Income (KDD). These experiments were instead completed on a Linux Cluster of Xeon $2.8 \mathrm{GHz}$ CPUs, an environment that does not allow reliable time measurements to be taken. For RF10 and RF100 on Covertype, compute times were estimated by averaging over those runs that could be completed on the virtual machine. No runs could be completed on the virtual machine for RF100 on Census-Income (KDD) and so no time results are reported.
} 

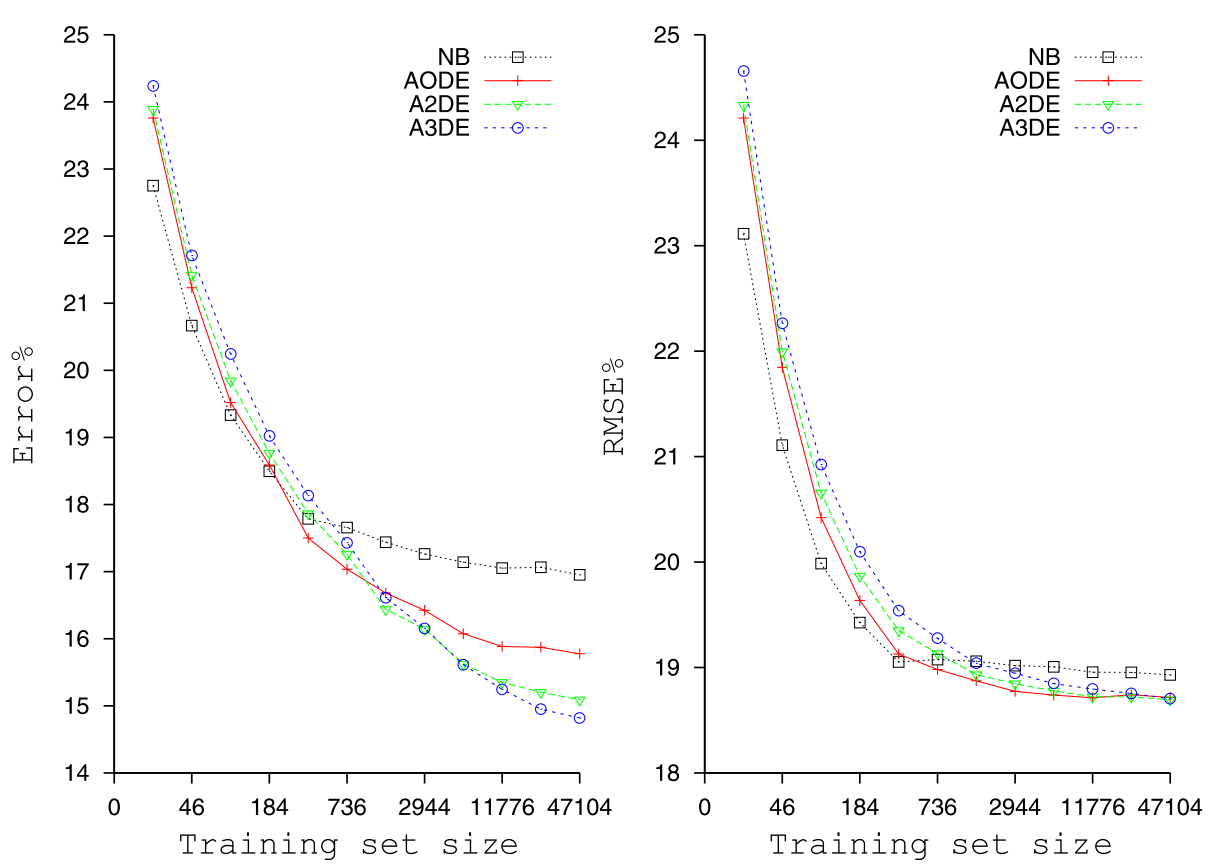

Fig. 2 Zero-one loss and RMSE of NB and $\mathrm{A} n \mathrm{DE}$ on Adult dataset, as function of training set size

by how well the two algorithms' learning biases match the underlying distribution, by their variance, and by the quantity of data. A low variance algorithm will usually have an advantage for small data while a low bias algorithm will usually be advantaged by large data. For our datasets, both AODE and A2DE reduce both zero-one loss and RMSE significantly often relative to NB. While A2DE obtains lower zero-one loss and RMSE than AODE more often than the reverse, this difference is not found to be significant.

To investigate in greater detail our expectation that algorithms with lower variance will be advantaged for small data and those with lower bias for larger data, we form learning curves for Adult, replicating the method of Webb et al. (2005). 1000 objects are selected at random as a test set and training sets were sampled from the remaining objects. The training set size starts from 23 and then doubles up to 47104, this being a progression that ends with as close to all the available data as possible once the 1000 test cases are removed. This process is repeated 50 times and each algorithm is evaluated on the resulting training-test set pairs. The learning curves of zero-one loss and RMSE for NB, AODE, A2DE and A3DE are presented in Fig. 2.

The plots for zero-one loss clearly show the predicted trade-off for increasing $n$. At the smallest data size, where low variance is more important than low bias, zero-one loss is minimized by $n=0(\mathrm{NB})$ and increases as $n$ increases. At the largest data size, where low bias is most important, this dimensionality is reversed. A similar trend is shown with respect to RMSE although the algorithms have not yet achieved their asymptotic rates at the largest data sizes available.

It is interesting to see how the relative bias/variance trade-offs of increasing $n$ play off when NB's attribute independence assumption holds. The LED dataset has a specific configuration of attribute-values for each class, making the attributes conditionally independent given the class. Each attribute has 10\% noise added. AODE and A2DE overfit this noise, 
Table 4 Win/draw/loss: AnDE, $n=0,1$ and 2 , on the ten largest data sets

\begin{tabular}{|c|c|c|c|c|c|c|}
\hline & \multicolumn{2}{|c|}{ A2DE vs AODE } & \multicolumn{2}{|c|}{ A2DE vs NB } & \multicolumn{2}{|c|}{ AODE vs NB } \\
\hline & $\mathrm{W} / \mathrm{D} / \mathrm{L}$ & $p$ & $\mathrm{~W} / \mathrm{D} / \mathrm{L}$ & $p$ & $\mathrm{~W} / \mathrm{D} / \mathrm{L}$ & $p$ \\
\hline Zero-ol & $10 / 0 / 0$ & 0.001 & $10 / 0 / 0$ & 0.001 & $10 / 0 / 0$ & 0.001 \\
\hline RMSE & $10 / 0 / 0$ & 0.001 & $10 / 0 / 0$ & 0.001 & $10 / 0 / 0$ & 0.001 \\
\hline
\end{tabular}
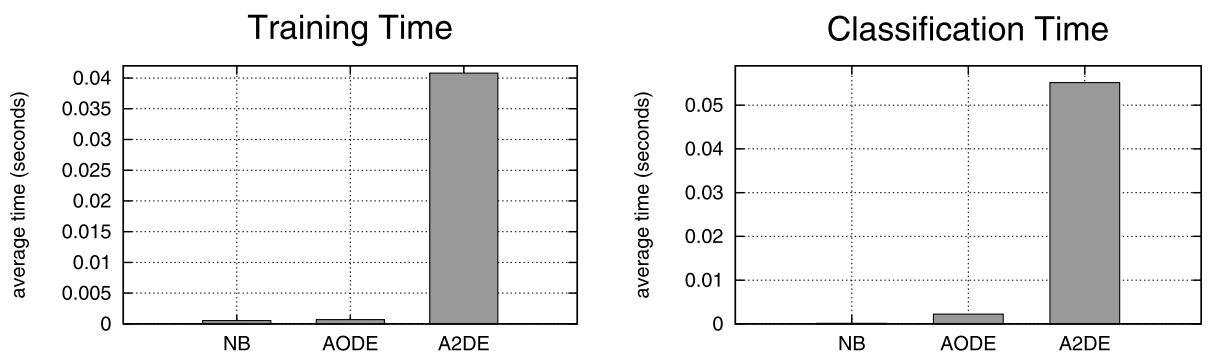

Fig. 3 Average per-example training and classification times for NB, AODE and A2DE

leading to increased error. NB's zero-one loss is 0.2627, AODE's is 0.2639 and A2DE's is 0.2667 . These outcomes are with training set sizes of 500. Using the UCI data generator, we generated 10 LED datasets comprising 2,000 and 10 comprising 4,000 instances and repeated the cross-validation experiments thereon. For the datasets of 2,000, the training set size is 1,000 and the mean and standard deviation of the respective zero-one loss is NB: $0.2603 \pm 0.0099$, AODE: $0.2601 \pm 0.0101$ and A2DE $0.2603 \pm 0.0102$. For the datasets of 4,000, the training set size is 2,000 and the mean and standard deviation of the respective zero-one loss is NB: $0.2597 \pm 0.0049$, AODE: $0.2598 \pm 0.0051$ and A2DE: $0.2603 \pm 0.0053$. It seems clear that increasing training set sizes rapidly reduces the error advantage that $\mathrm{NB}$ enjoys in this context where its conditional attribute assumption is satisfied.

As final confirmation that higher $n$ is best suited to larger data, on the ten largest datasets, those with more than 8,000 examples, A2DE always achieves lower zero-one loss and RMSE than AODE $(p=0.001)$, see Table 4 .

As expected, both training and classification compute times increase as $n$ increases. Figure 3 shows the grand averages for the per-example training and classification times for each algorithm.

\subsection{Comparison with TAN}

We here explore the relative benefits of discriminative search for a single best Bayesian classifier model against A $n$ DE's search-free approach of averaging over a class of Bayesian classifier models. To this end we compare AODE and A2DE with TAN. Table 5 presents win/draw/loss results comparing AODE and A2DE to TAN.

Overall, TAN has an advantage in bias but a disadvantage in variance relative to AODE. When using search to select a single 1-DBC model is compared to averaging over a class of 2-DBCs, the bias advantage is lost but the variance disadvantage remains. The relative bias-variance tradeoffs of AODE and TAN result in a general error advantage to AODE. Comparing TAN to A2DE, TAN no longer has a bias advantage, and at this higher value of $n$, the error advantage of the $\mathrm{A} n \mathrm{DE}$ classifier becomes even more consistent.

Figure 4 shows the relative training and classification times for AODE, A2DE and TAN. It is clear that $\mathrm{A} 2 \mathrm{DE}$ has a considerably greater computational requirements both for training 
Table 5 Win/draw/loss: AnDE, $n=1$ and 2 vs TAN on all 62 data sets

\begin{tabular}{llrllr}
\hline & \multicolumn{2}{l}{ A2DE vs TAN } & & \multicolumn{2}{l}{ AODE vs TAN } \\
\cline { 2 - 3 } \cline { 6 - 6 } & W $/ \mathrm{D} / \mathrm{L}$ & $p$ & & W $/ \mathrm{D} / \mathrm{L}$ & \multicolumn{1}{c}{$p$} \\
\hline Bias & $34 / 0 / 28$ & 0.263 & & $20 / 1 / 41$ & $\mathbf{0 . 0 0 5}$ \\
Variance & $48 / 0 / 14$ & $<\mathbf{0 . 0 0 1}$ & & $52 / 1 / 9$ & $<\mathbf{0 . 0 0 1}$ \\
Zero-one loss & $48 / 0 / 14$ & $<\mathbf{0 . 0 0 1}$ & & $43 / 1 / 18$ & $\mathbf{0 . 0 0 1}$ \\
RMSE & $43 / 1 / 18$ & $\mathbf{0 . 0 0 1}$ & & $40 / 1 / 21$ & $\mathbf{0 . 0 1 0}$ \\
\hline
\end{tabular}
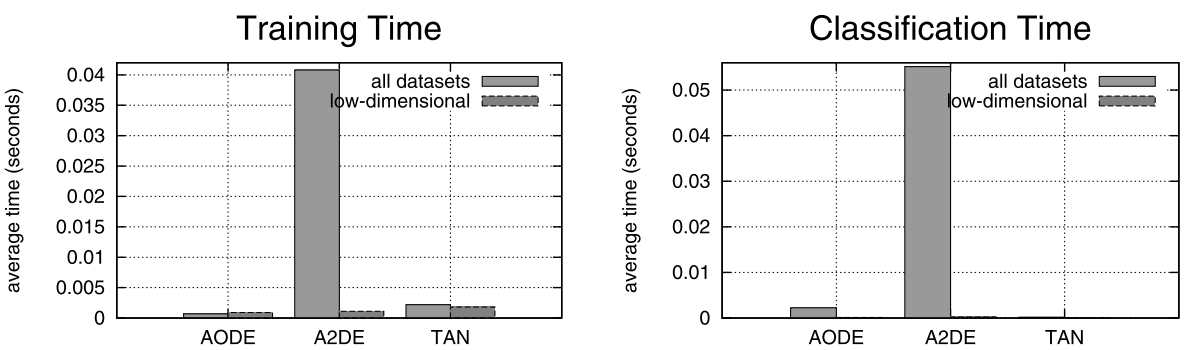

Fig. 4 Average per-example training and classification times for AODE, A2DE and TAN. (Two values are shown for each algorithm, the average across all datasets and the average across the ten lowest-dimensional (4-7 attributes) datasets)

Table 6 Win/draw/loss: AnDE, $n=1$ and 2 vs MAPLMG on all 62 data sets

\begin{tabular}{llllll}
\hline & \multicolumn{2}{l}{ A2DE vs MAPLMG } & & \multicolumn{2}{l}{ AODE vs MAPLMG } \\
\cline { 2 - 3 } & W/D $/ \mathrm{L}$ & $p$ & & W $/ \mathrm{D} / \mathrm{L}$ & $p$ \\
\hline Bias & $40 / 0 / 22$ & $\mathbf{0 . 0 1 5}$ & & $17 / 4 / 41$ & $\mathbf{0 . 0 0 1}$ \\
Variance & $19 / 1 / 42$ & $\mathbf{0 . 0 0 2}$ & & $36 / 5 / 21$ & $\mathbf{0 . 0 3 1}$ \\
Zero-one loss & $30 / 1 / 31$ & 0.500 & & $22 / 4 / 36$ & $\mathbf{0 . 0 4 3}$ \\
RMSE & $34 / 1 / 28$ & 0.263 & & $19 / 0 / 39$ & $\mathbf{0 . 0 0 6}$ \\
\hline
\end{tabular}

and classification. However, this disadvantage disappears when we consider only the ten lowest dimensional datasets, also illustrated in this figure.

\subsection{Comparison with MAPLMG}

As discussed above, we wish to investigate the relative payoffs obtained by investing additional computation to that required by AODE by respectively using discriminative learning of weights or, alternatively, increasing the dimensionality of the probabilities from which the posterior probability is extrapolated. To this end, Table 6 presents win/draw/loss results comparing A2DE and AODE to MAPLMG.

As established by previous research (Cerquides and Mántaras 2005), MAPLMG's approach of using discriminative learning of weights for the AODE linear combination significantly reduces bias relative to AODE at the cost of an increase in variance. However, relative to this discriminative approach to extrapolating from three-dimensional probabilities, A2DE's search-free approach to extrapolating from four-dimensional probabilities further reduces bias at the cost of an increase in variance. While the resulting difference in error is not found to be significant across the full suite of 62 datasets, when the ten largest datasets 
Table 7 Win/draw/loss: AnDE, $n=1$ and 2 vs MAPLMG on the ten largest data sets

\begin{tabular}{llllll}
\hline & \multicolumn{2}{l}{ A2DE vs MAPLMG } & & \multicolumn{2}{l}{ AODE vs MAPLMG } \\
\cline { 2 - 3 } & W/D/L & $p$ & & W/D/L & $p$ \\
\hline Zero-one loss & $10 / 0 / 0$ & $\mathbf{0 . 0 0 1}$ & & $1 / 1 / 8$ & $\mathbf{0 . 0 3 9}$ \\
RMSE & $10 / 0 / 0$ & $\mathbf{0 . 0 0 1}$ & & $0 / 0 / 10$ & $\mathbf{0 . 0 0 1}$ \\
\hline
\end{tabular}

Training Time

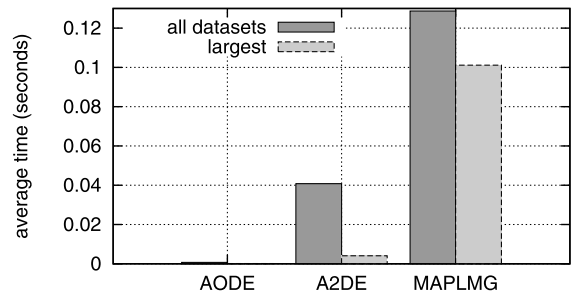

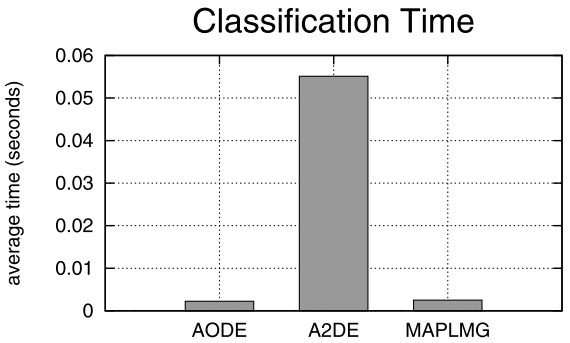

Fig. 5 Average per-example training and classification times for AODE, A2DE and MAPLMG. (In addition to the times for all datasets, training times are shown for the ten largest datasets)

are considered, the lower bias algorithm, A2DE, consistently achieves lower zero-one loss and RMSE than MAPLMG ( $p=0.001)$ (see Table 7).

MAPLMG's Bayesian model averaging comes at considerable cost in training time. Figure 5 shows the average per-example training and test times for AODE, A2DE and MAPLMG. Note that MAPLMG is implemented as an external function to Weka, and hence is likely to be inherently more efficient. The training and test times include a substantial fixed overhead, and hence the per-instance training times should decrease if the complexity is linear with respect to the training set size. However, MAPLMG's super-linear training complexity minimizes this effect, demonstrating that it will not be feasible to apply it to very large data.

\subsection{Comparison with Feating}

To understand how the AnDE approach performs relative to Feating NB, we compare A2DE, that calculates the mean of the joint probabilities, with a variant PA2DE, that calculates the mean of the posterior probabilities, and another variant FA2DE, that calculates the mode of the classes predicted by the submodels. As described in Sect. 3 and the start of Sect. 4, these embody the two main differences between AnDE and Feating NB.

Table 8 shows the win/draw/loss results comparing A2DE to these variants. It is clear that both variants have higher bias but lower variance than A2DE. It is straightforward to understand why Feating would have lower variance. The mode is a much more stable estimator of central tendency than the mean, which can be greatly influenced by a single outlier. It is less obvious why lower variance should result from averaging over the estimates of the posterior rather than of the joint probability. Nonetheless, the result is consistent with Cerquides and de Màntaras' (2005) finding that a linear combination of joint probability estimates resulted in higher accuracy than a linear combination of posterior probability estimates. This remains an interesting unexplained phenomena worthy of further investigation.

Over the full range of datasets these differences in bias and variance profiles do not result in statistically significant differences on either measure of error, except with respect 
Table 8 Win/draw/loss: A2DE vs PA2DE and FA2DE

\begin{tabular}{llrllr}
\hline & \multicolumn{2}{l}{ A2DE vs PA2DE } & & \multicolumn{2}{l}{ A2DE vs FA2DE } \\
\cline { 2 - 3 } \cline { 6 - 7 } & W/D/L & $p$ & & W $/ \mathrm{D} / \mathrm{L}$ & \multicolumn{1}{l}{$p$} \\
\hline Bias & $41 / 2 / 19$ & $<\mathbf{0 . 0 0 1}$ & & $46 / 1 / 15$ & $<\mathbf{0 . 0 0 1}$ \\
Variance & $21 / 3 / 38$ & $\mathbf{0 . 0 1 8}$ & & $22 / 1 / 39$ & $\mathbf{0 . 0 2 0}$ \\
Zero-one loss & $33 / 1 / 28$ & 0.304 & & $36 / 2 / 24$ & $\mathbf{0 . 0 7 8}$ \\
RMSE & $30 / 0 / 32$ & 0.449 & & $49 / 0 / 13$ & $<\mathbf{0 . 0 0 1}$ \\
\hline
\end{tabular}

\begin{tabular}{llllll}
\hline & \multicolumn{2}{l}{ A2DE vs PA2DE } & & \multicolumn{2}{l}{ A2DE vs FA2DE } \\
\cline { 2 - 3 } \cline { 5 - 6 } W/D/L & $p$ & & W/D/L & $p$ \\
\hline Zero-one loss & $8 / 0 / 2$ & 0.109 & & $9 / 0 / 1$ & $\mathbf{0 . 0 2 1}$ \\
RMSE & $8 / 0 / 2$ & 0.109 & & $8 / 1 / 1$ & $\mathbf{0 . 0 3 9}$ \\
\hline
\end{tabular}

Table 9 Win/draw/loss: A2DE vs PA2DE and FA2DE on the ten largest datasets 
Table 10 Win/draw/loss: A $n \mathrm{DE}, n=0,1$ and 2, vs RF10 and RF100 on all 62 data sets

\begin{tabular}{|c|c|c|c|c|c|}
\hline & & \multicolumn{2}{|c|}{$\mathrm{A} n \mathrm{DE}$ vs RF10 } & \multicolumn{2}{|c|}{ A $n$ DE vs RF100 } \\
\hline & & $\mathrm{W} / \mathrm{D} / \mathrm{L}$ & $p$ & $\overline{\mathrm{W} / \mathrm{D} / \mathrm{L}}$ & $p$ \\
\hline \multirow{4}{*}{ 崩 } & Bias & $18 / 1 / 43$ & 0.001 & $22 / 2 / 38$ & 0.026 \\
\hline & Variance & $57 / 0 / 5$ & $<0.001$ & $45 / 1 / 16$ & $<0.001$ \\
\hline & Zero-one loss & $42 / 0 / 20$ & 0.004 & $36 / 3 / 23$ & 0.059 \\
\hline & RMSE & $40 / 0 / 22$ & 0.015 & $35 / 0 / 27$ & 0.187 \\
\hline \multirow{4}{*}{ 崩 } & Bias & $16 / 0 / 46$ & $<0.001$ & $20 / 0 / 42$ & 0.004 \\
\hline & Variance & $57 / 1 / 4$ & $<0.001$ & $47 / 0 / 15$ & $<0.001$ \\
\hline & Zero-one loss & $41 / 0 / 21$ & 0.008 & $33 / 1 / 28$ & 0.304 \\
\hline & RMSE & $39 / 0 / 23$ & 0.028 & $34 / 0 / 28$ & 0.263 \\
\hline \multirow{4}{*}{$\vec{z}$} & Bias & $14 / 1 / 47$ & $<0.001$ & $16 / 1 / 45$ & $<0.001$ \\
\hline & Variance & $56 / 0 / 6$ & $<0.001$ & $51 / 0 / 11$ & $<0.001$ \\
\hline & Zero-one loss & $33 / 0 / 29$ & 0.352 & $30 / 1 / 31$ & 0.500 \\
\hline & RMSE & $30 / 0 / 32$ & 0.450 & $28 / 0 / 34$ & 0.263 \\
\hline
\end{tabular}

Table 11 Win/draw/loss: A $n \mathrm{DE}, n=0,1$ and 2, vs RF10 and RF100 on the ten largest data sets

\begin{tabular}{|c|c|c|c|c|c|}
\hline & & \multicolumn{2}{|c|}{ A $n \mathrm{DE}$ vs RF 10} & \multicolumn{2}{|c|}{ A $n$ DE vs RF100 } \\
\hline & & $\mathrm{W} / \mathrm{D} / \mathrm{L}$ & $p$ & $\mathrm{~W} / \mathrm{D} / \mathrm{L}$ & $p$ \\
\hline \multirow{2}{*}{ 䆥 } & Zero-one loss & $2 / 0 / 8$ & 0.055 & $1 / 1 / 8$ & 0.020 \\
\hline & RMSE & $3 / 0 / 7$ & 0.172 & $2 / 0 / 8$ & 0.055 \\
\hline \multirow{2}{*}{ 荇 } & Zero-one loss & $0 / 0 / 10$ & 0.001 & $0 / 0 / 10$ & 0.001 \\
\hline & RMSE & $1 / 0 / 9$ & 0.011 & $0 / 0 / 10$ & 0.001 \\
\hline \multirow{2}{*}{$\bar{z}$} & Zero-one loss & $0 / 0 / 10$ & 0.001 & $0 / 0 / 10$ & 0.001 \\
\hline & RMSE & $0 / 0 / 10$ & 0.001 & $0 / 0 / 10$ & 0.001 \\
\hline
\end{tabular}

for very large training data, in the absence of any prior knowledge of the nature of the multivariate probability distribution that the data embodies, Random Forests are likely to achieve lower error than an AnDE classifier, although the data quantity at which this is achieved will be ever greater as the dimensionality of $\mathrm{A} n \mathrm{DE}$ is increased.

However, Random Forests' error advantage for large data comes at a cost in training time. Figure 6 shows the training and classification times for AODE, A2DE, RF10 and RF100. It is apparent that, overall, RF100 has very high training times. While A2DE's training time does approach RF100's for high dimensional data, for small data and low dimensional data its training times are competitive with RF10. On the other hand, A2DE requires substantially more classification time on average than Random Forests. This requirement grows greatly with high-dimensional data. A2DE will not be feasible for classification of large numbers of high-dimensional objects. In contrast, its classification time is very competitive on lowdimensional data.

\section{Conclusions and directions for future research}

A $n$ DE provides an attractive framework for developing machine learning techniques. A single parameter $n$ controls a bias-variance trade-off such that $n=a$ provides a classifier whose 

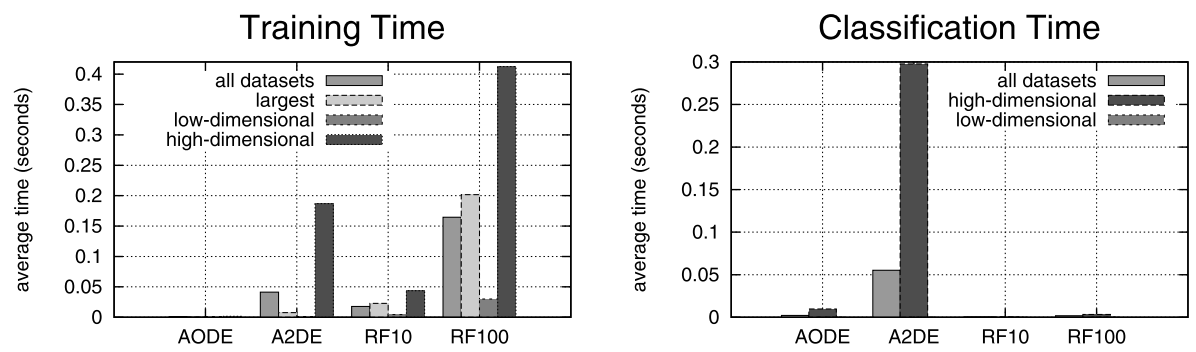

Fig. 6 Average per-example training and classification times for AODE, A2DE, RF10 and RF100. (Training times are presented for all, the ten largest (excluding Census Income, for which RF100 could not be executed on a machine for which reliable times could be obtained, thus 5,620-581,012 examples), the ten lowest dimensional (5-7 attributes) and the ten highest dimensional (43-70 attributes) datasets. Classification times are presented for all, the ten lowest dimensional and the ten highest dimensional datasets)

asymptotic error is the Bayes optimal error rate. However, for high-dimensional data only very low-dimensional forms of $\mathrm{A} n \mathrm{DE}$ are feasible. Nonetheless, we have established that higher-dimensional variants are likely to deliver greater accuracy than lower-dimensional alternatives when the number of training examples is high. In consequence, a promising direction for future research is to develop computationally efficient techniques for approximating $\mathrm{A} n \mathrm{DE}$ for high values of $n$.

A further unresolved issue is how to select an appropriate value of $n$ for any specific dataset $\mathcal{T}$. Are there more computationally efficient approaches than a simple wrapper-based comparison of each possible value?

A number of techniques have been developed for extending AODE to handle numeric data (Flores et al. 2009). There is a need to extend this work to the more general AnDE framework.

We have presented a strategy for learning without fitting the full multivariate probability distribution. We do not argue, however, that fitting the full multivariate probability distribution should necessarily be avoided. Indeed, it has been demonstrated that it is possible to reduce the error of AODE both by appropriate feature selection (Zheng and Webb 2006, 2007; Yang et al. 2007) and weighting of the submodels (Cerquides and Mántaras 2005; Jiang and Zhang 2006; Yang et al. 2007) in order to better fit the full multivariate probability distribution. Therefore, it is likely to be worthwhile to explore efficient methods for each of these strategies for higher values of $n$. If fast classification is required, and time for training is less constrained, approaches that use search to select a small number of submodels from an AnDE model are likely to be desirable. Where there is sufficient training time available, search for appropriate submodel weights is also likely to be useful.

We have developed a generative learning algorithm that generalizes the principles that underlie AODE to ever higher levels of dimensionality. It has the following desirable features:

- both time and space complexity are linear with respect to the number of training examples;

- it learns in a single pass through the training data;

- it performs direct prediction of class probabilities;

- it has integrated handling of missing values;

- it is robust in the face of noise;

- other than the choice of which instantiation (choice of $n$ ) and choice of smoothing technique, the approach uses no tunable parameters;

- it does not perform model selection; 
- a simple mechanism controls the bias/variance trade-off;

- it supports incremental learning;

- learning and classification can readily utilize parallel computation; and

- there is a direct theoretical basis that provides optimal prediction except insofar as clearly specified assumptions are violated.

A single parameter $n$ provides control over a bias-variance trade-off, such that higher values of $n$ are appropriate for greater numbers of training cases. AnDE demonstrates that it is possible to develop competitive learners without using search. Of further interest, this family of algorithms show that it is possible to develop low bias algorithms in a generative framework. Finally, A2DE proves to be a computationally tractable version of A $n \mathrm{DE}$ that delivers strong classification accuracy for large data without any parameter tuning.

Acknowledgements This research has been supported by Australian Research Council grant DP110101427. We are grateful to Mark Carman, Joao Gama, Kevin Korb and Nayyar Zaidi for insightful discussions on this research and feedback on drafts of this paper.

\section{Appendix: Detailed results}

Detailed results for Bias, Variance, zero-one Loss, RMSE, Training Time and Classification Times are presented in Tables 8 to 13. The datasets are listed in ascending order on number of instances. 


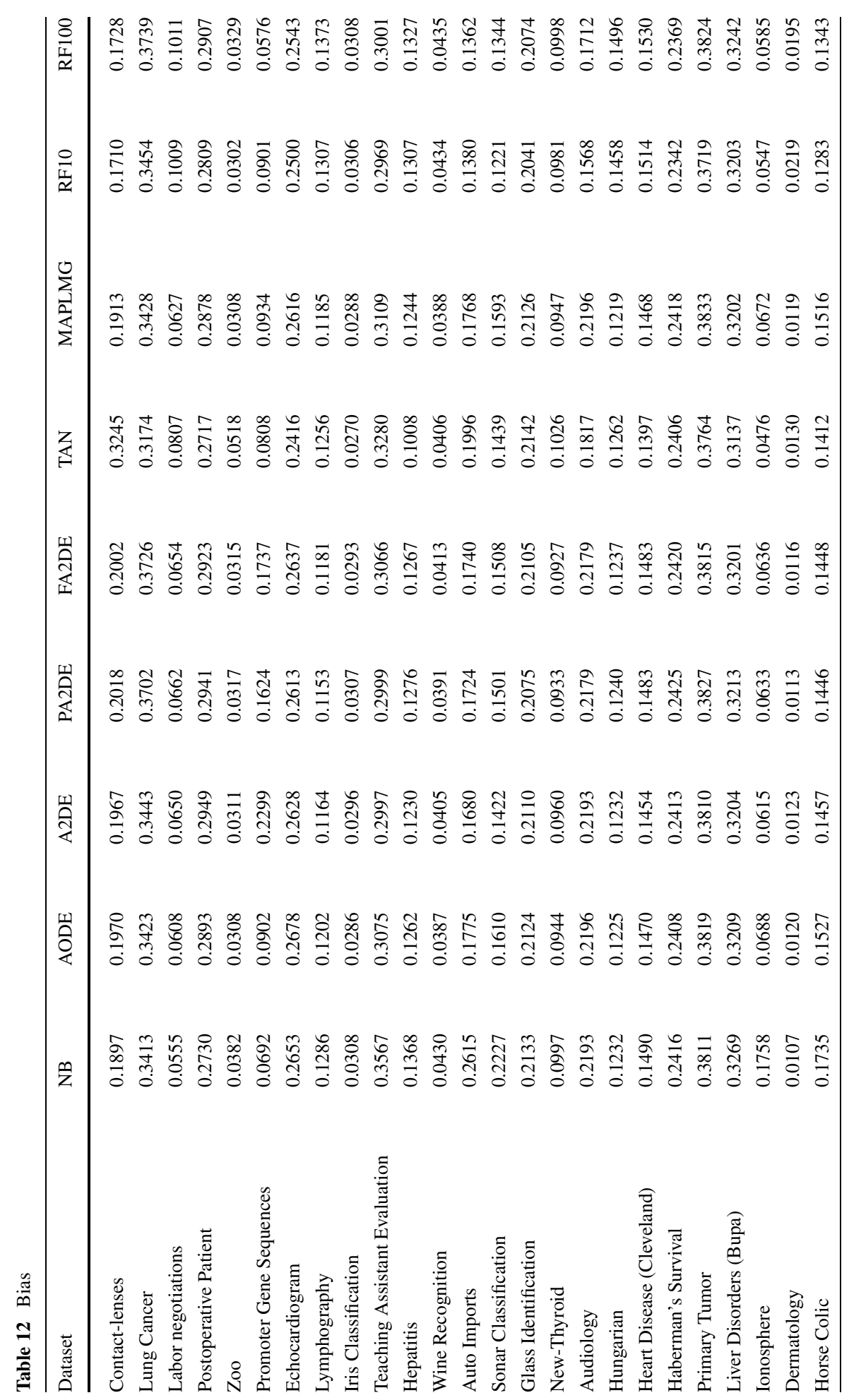




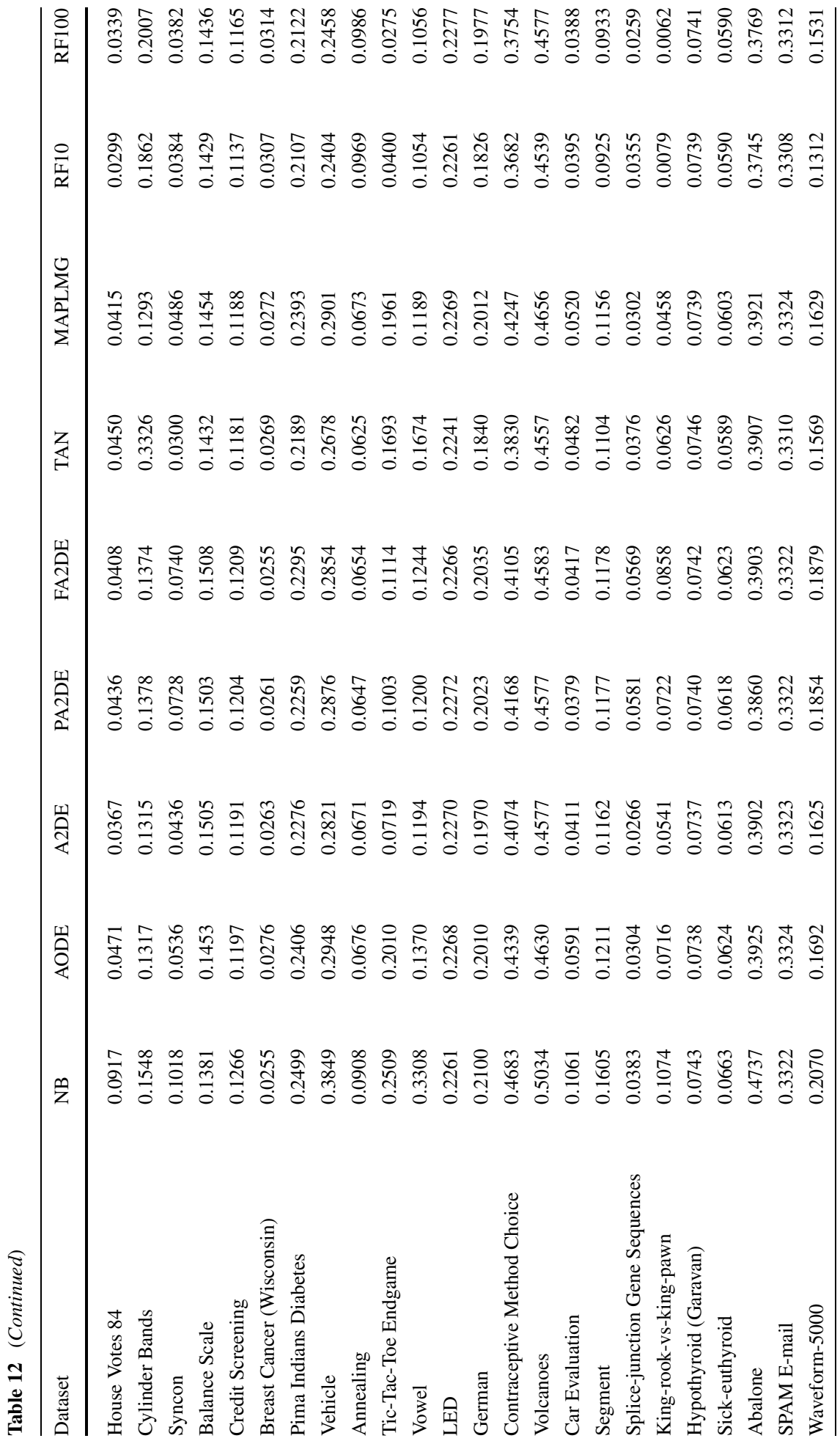




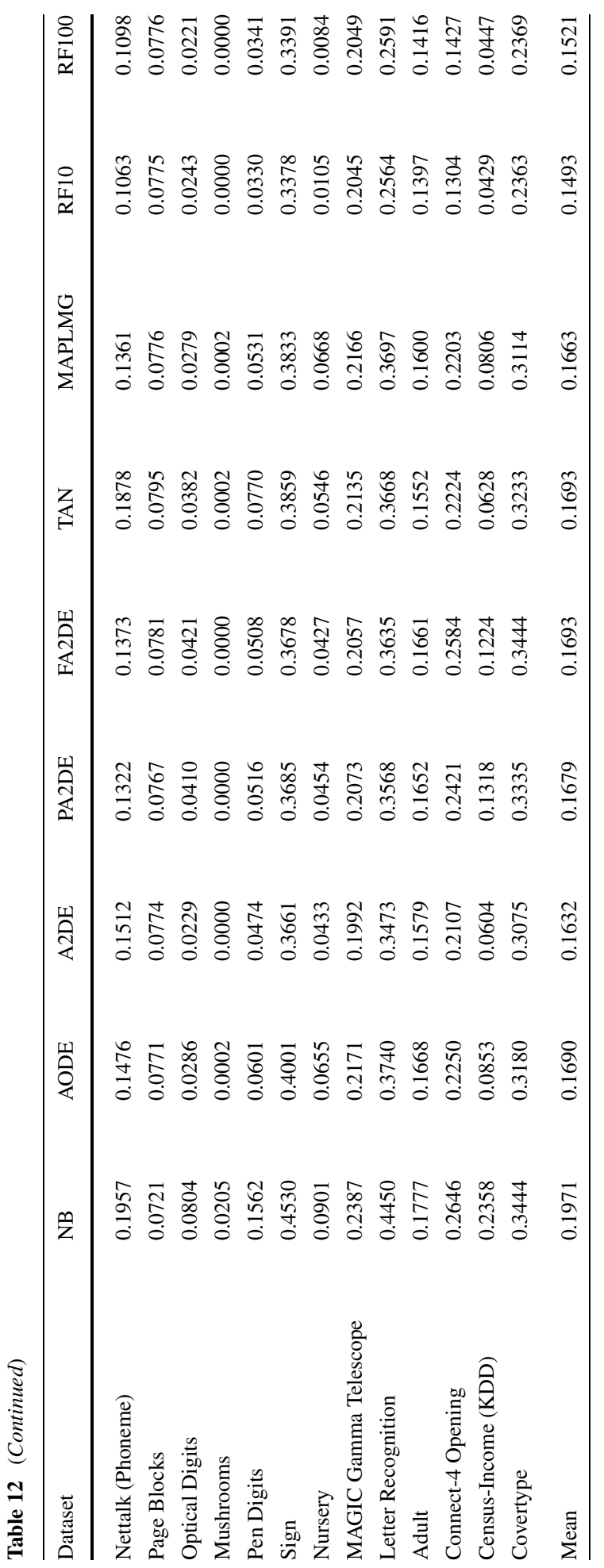




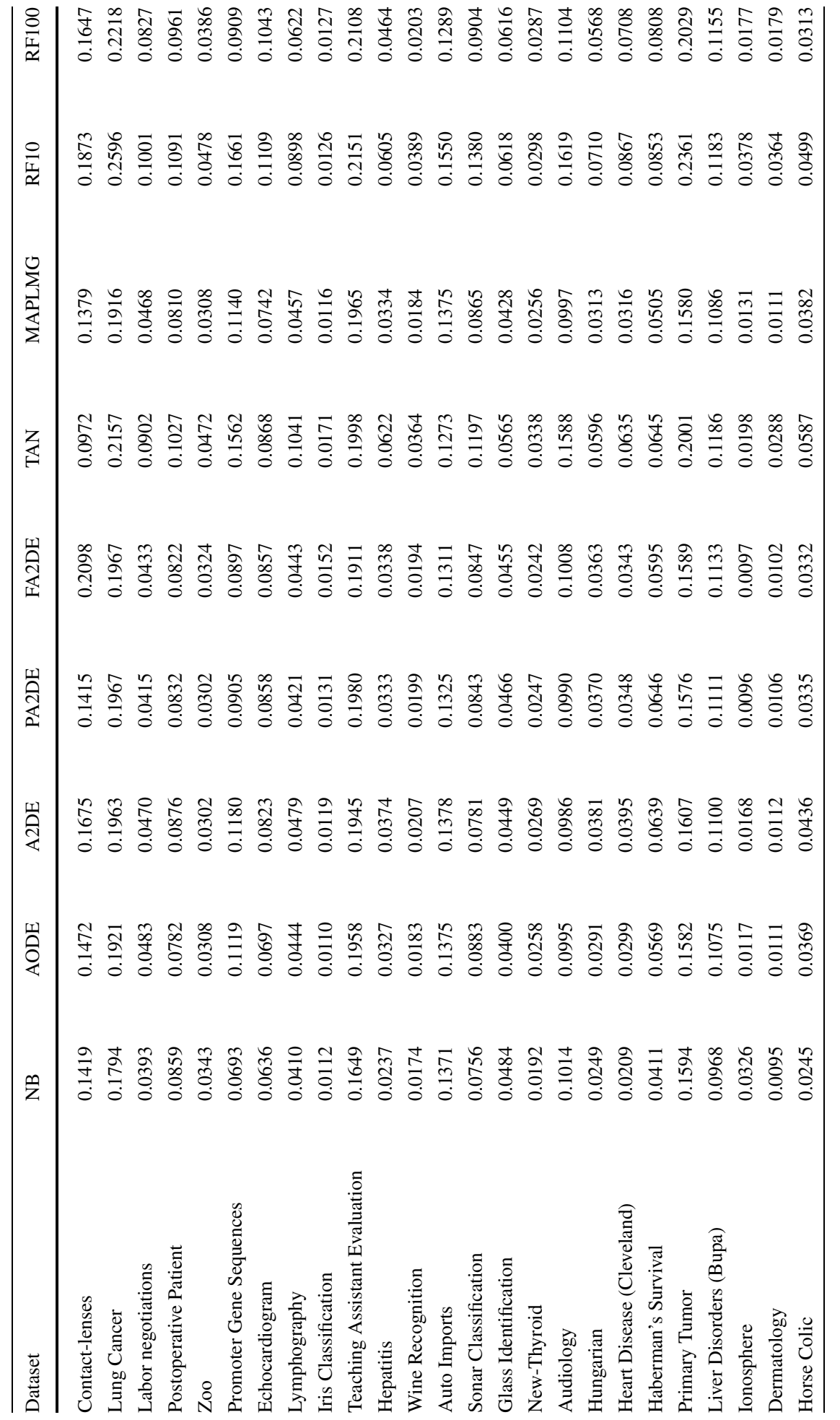




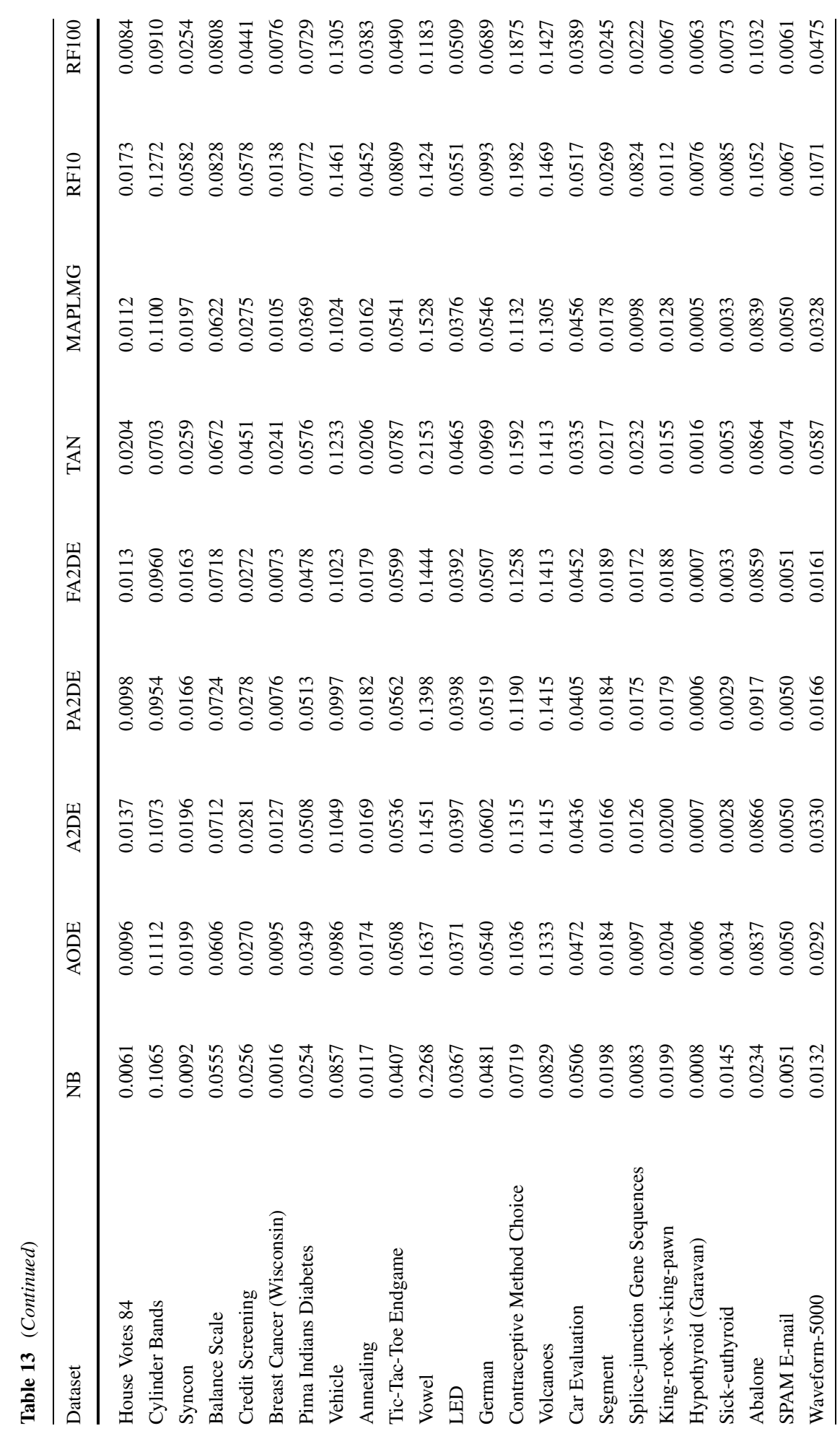




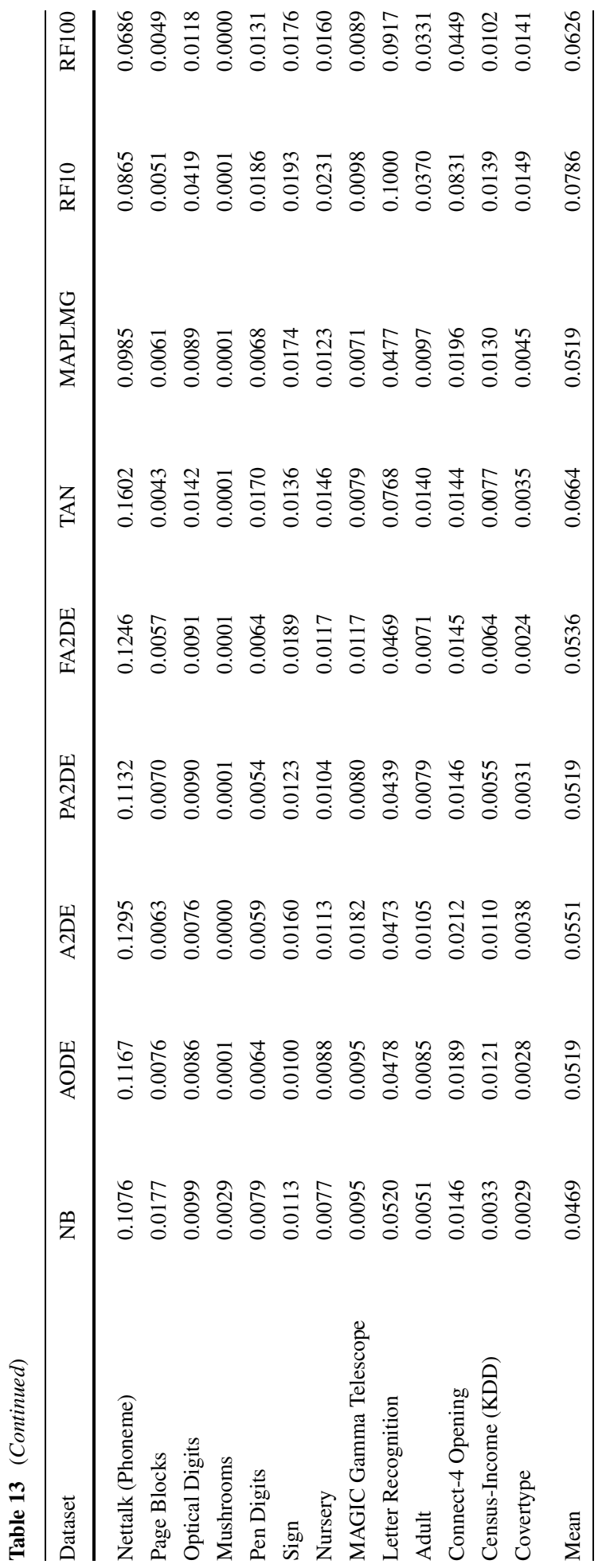




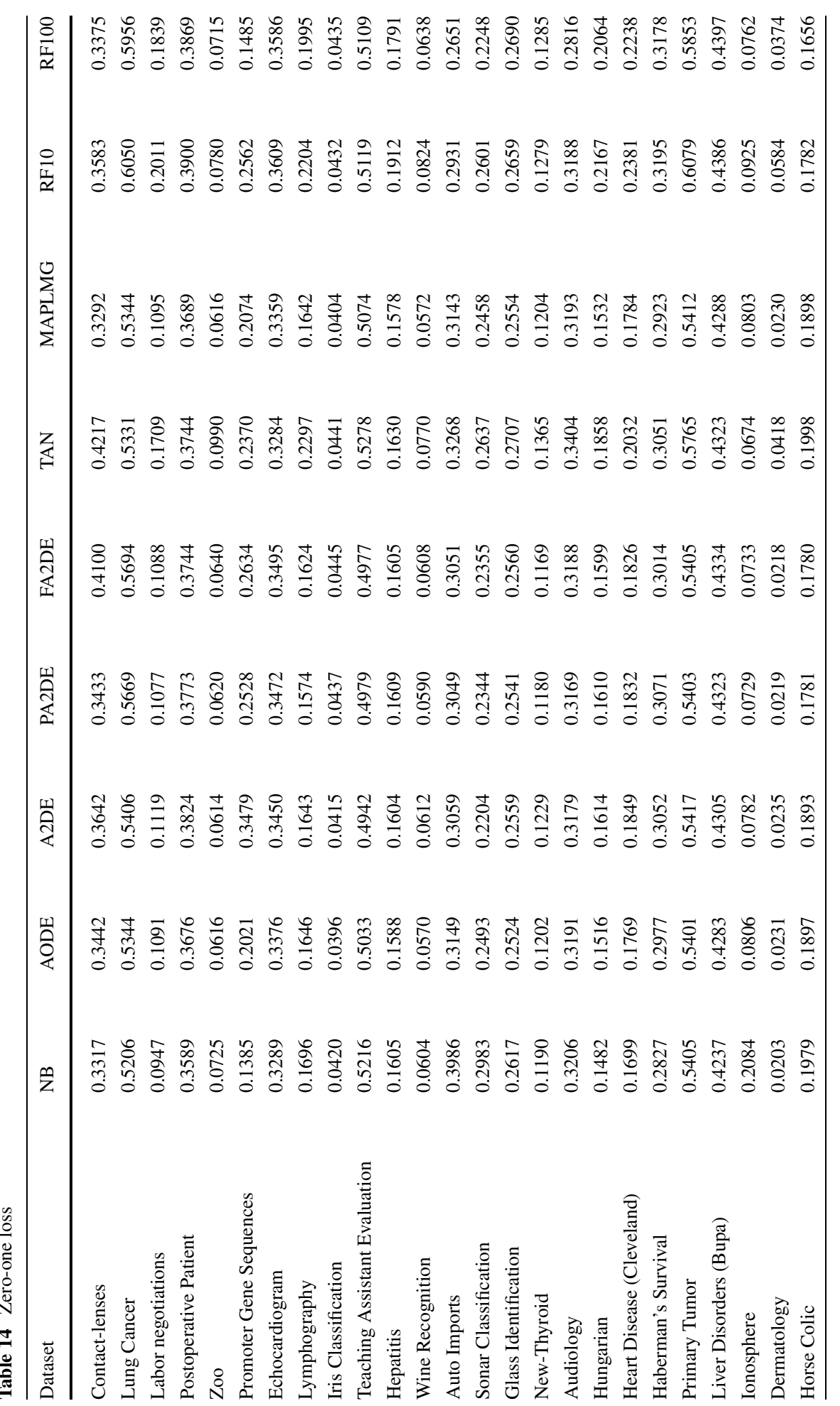




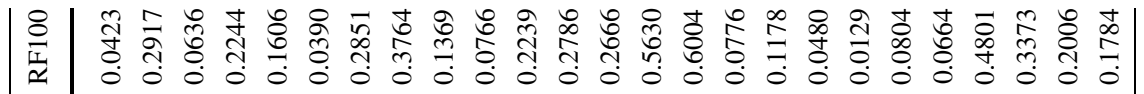

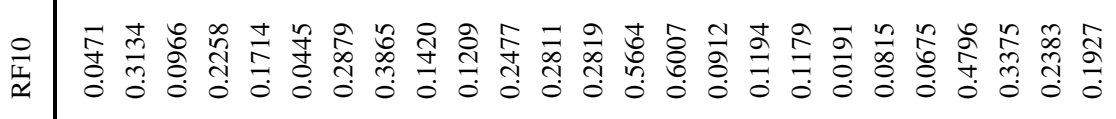

సิ ๙ิ 它

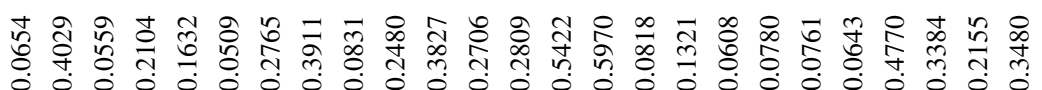

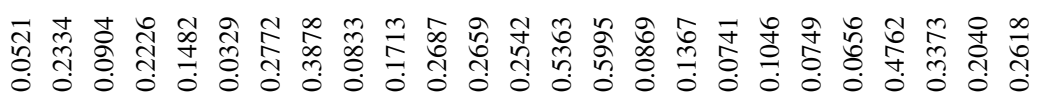

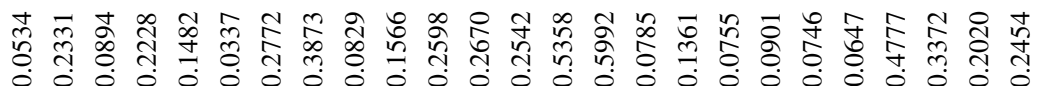

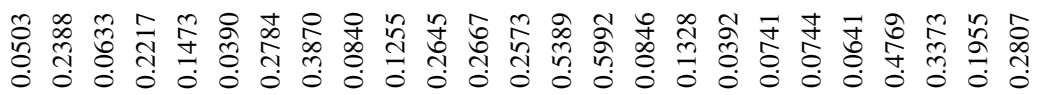

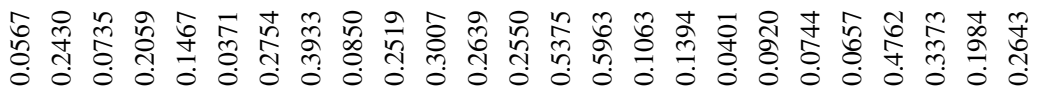

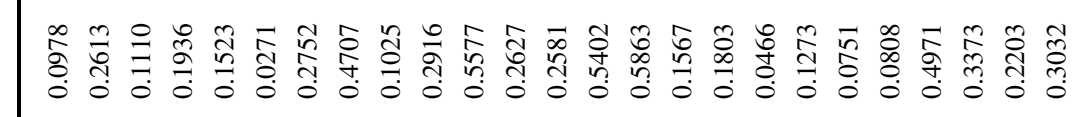
(n)

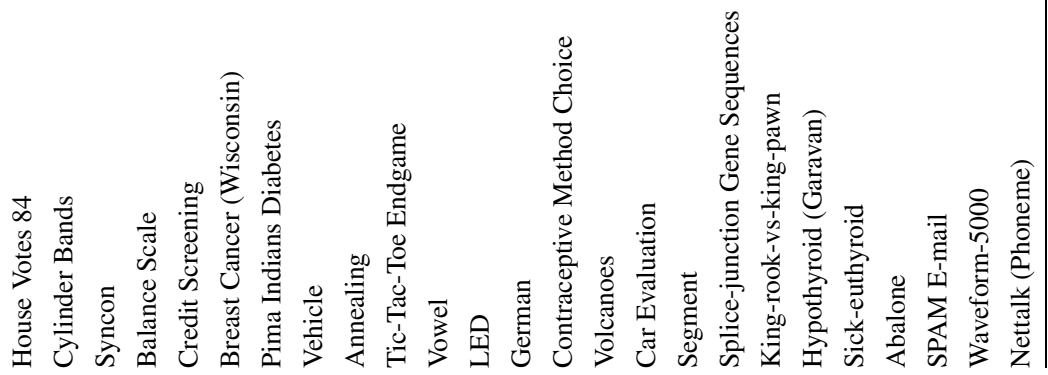




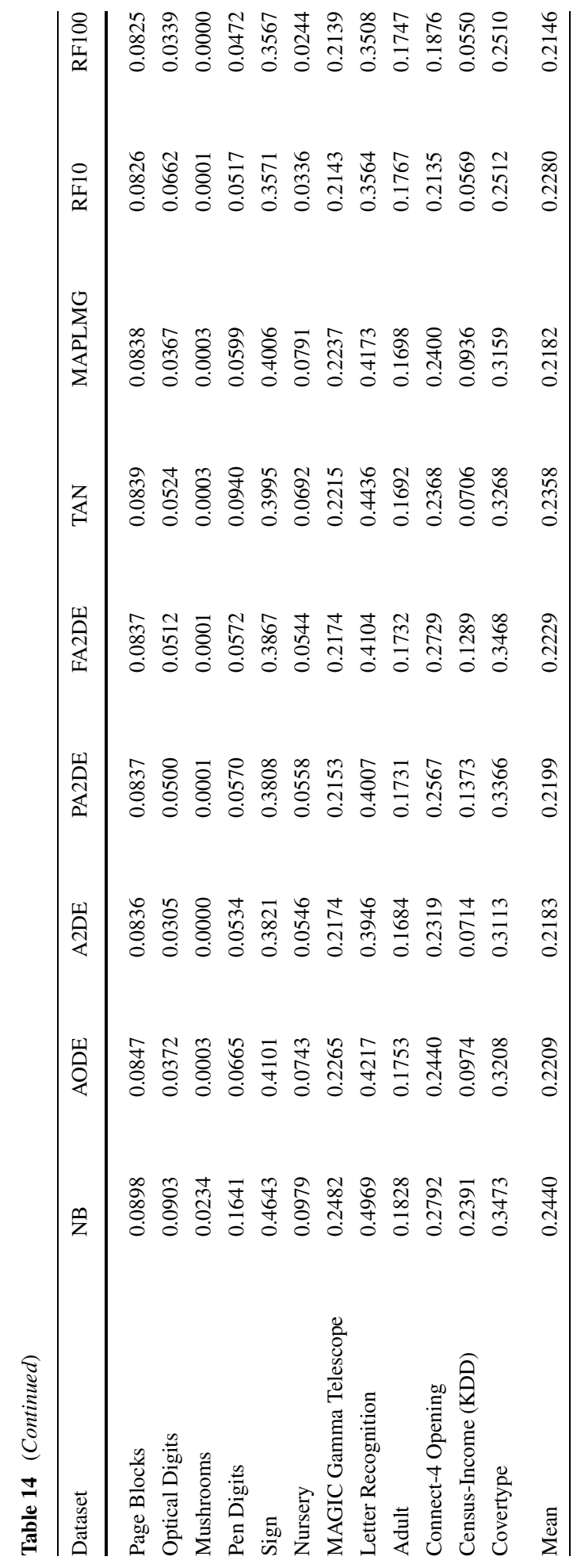




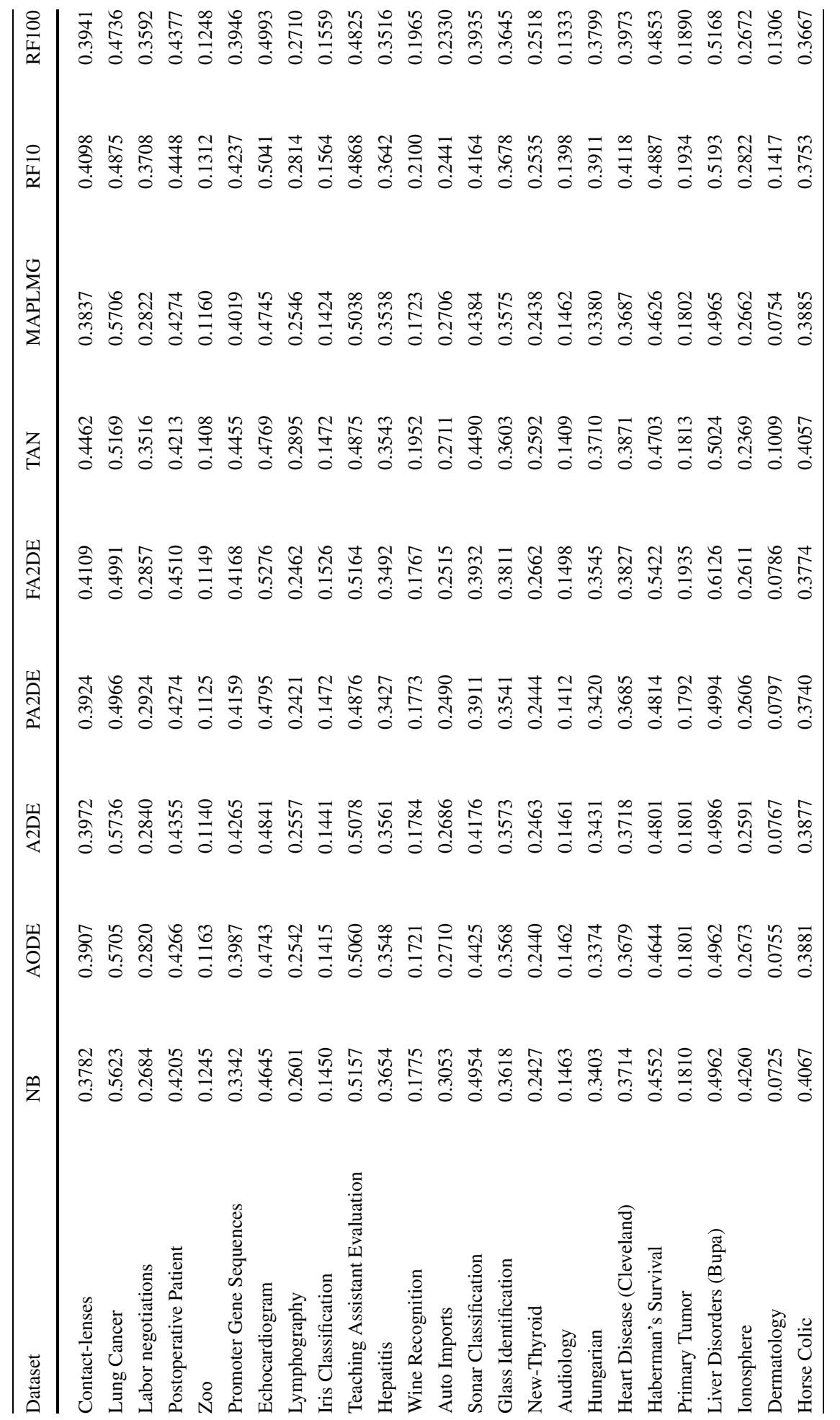




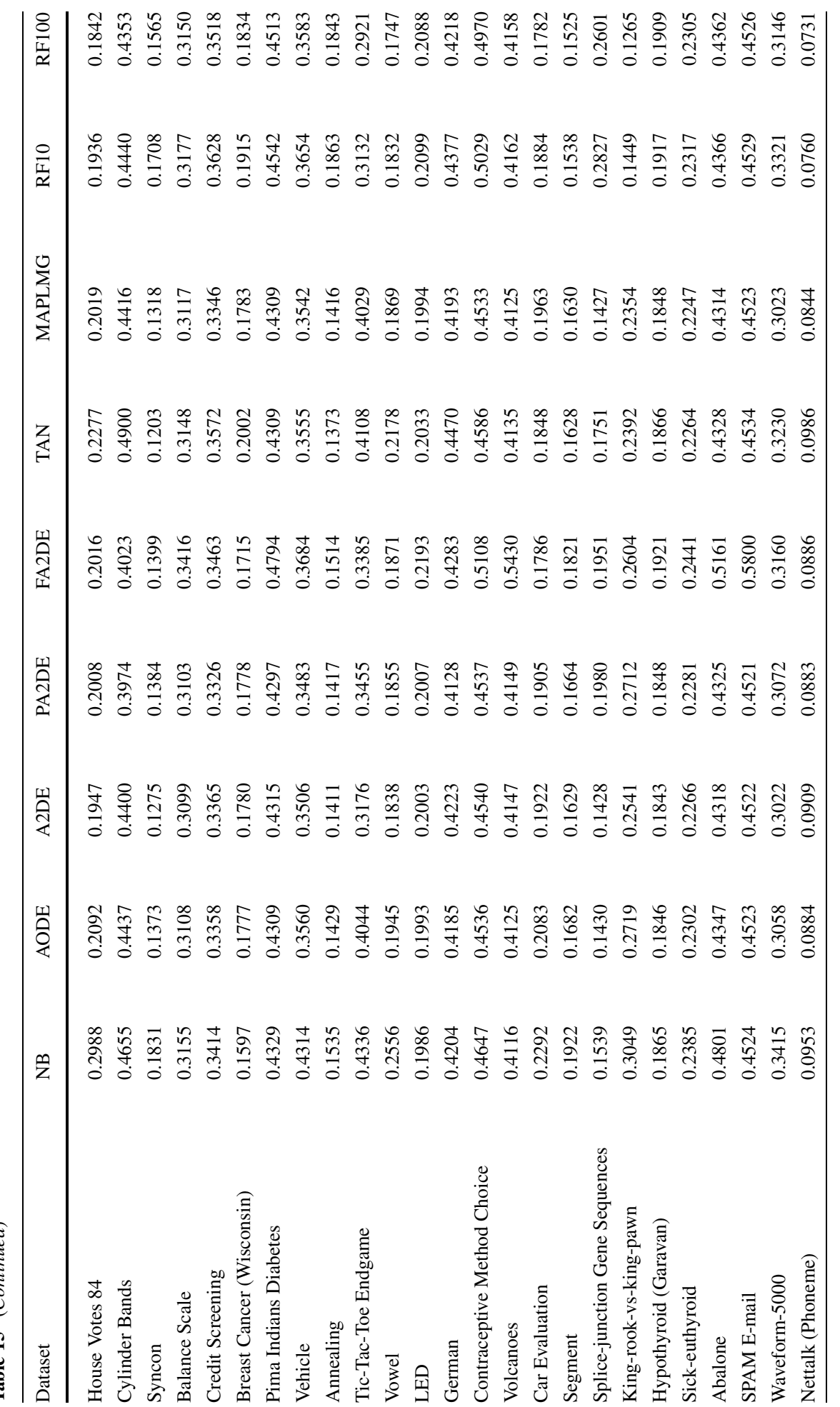




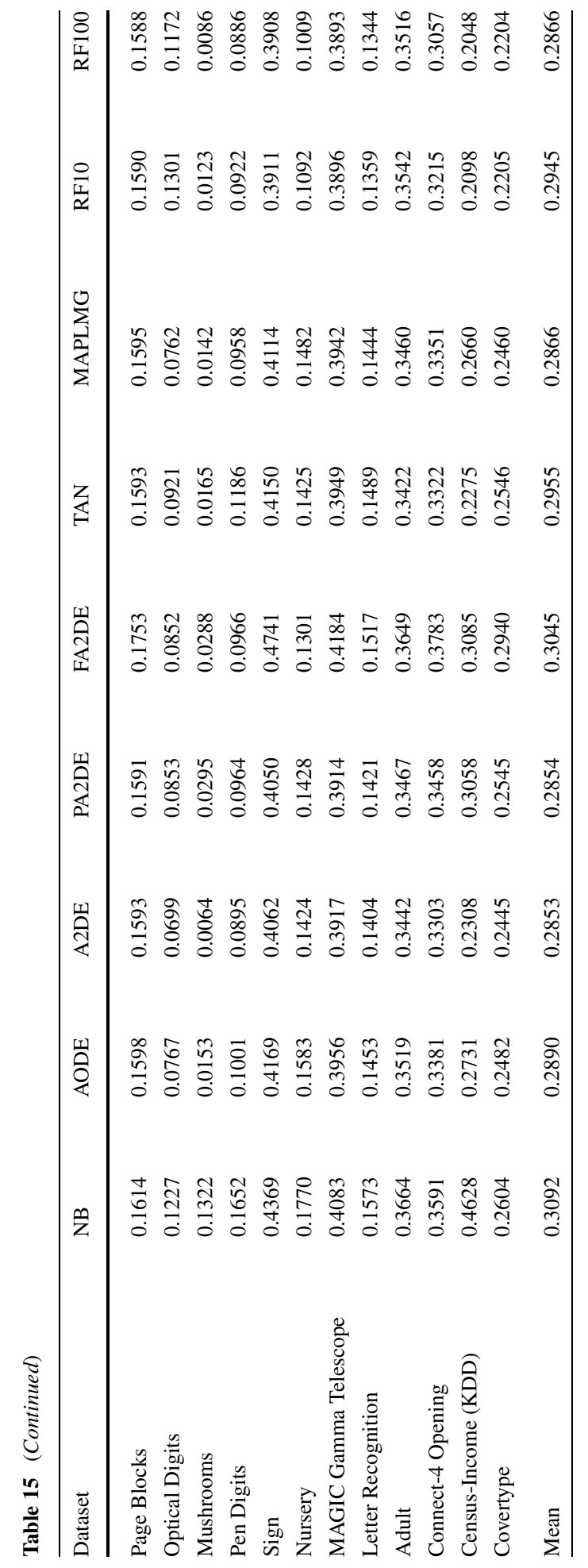




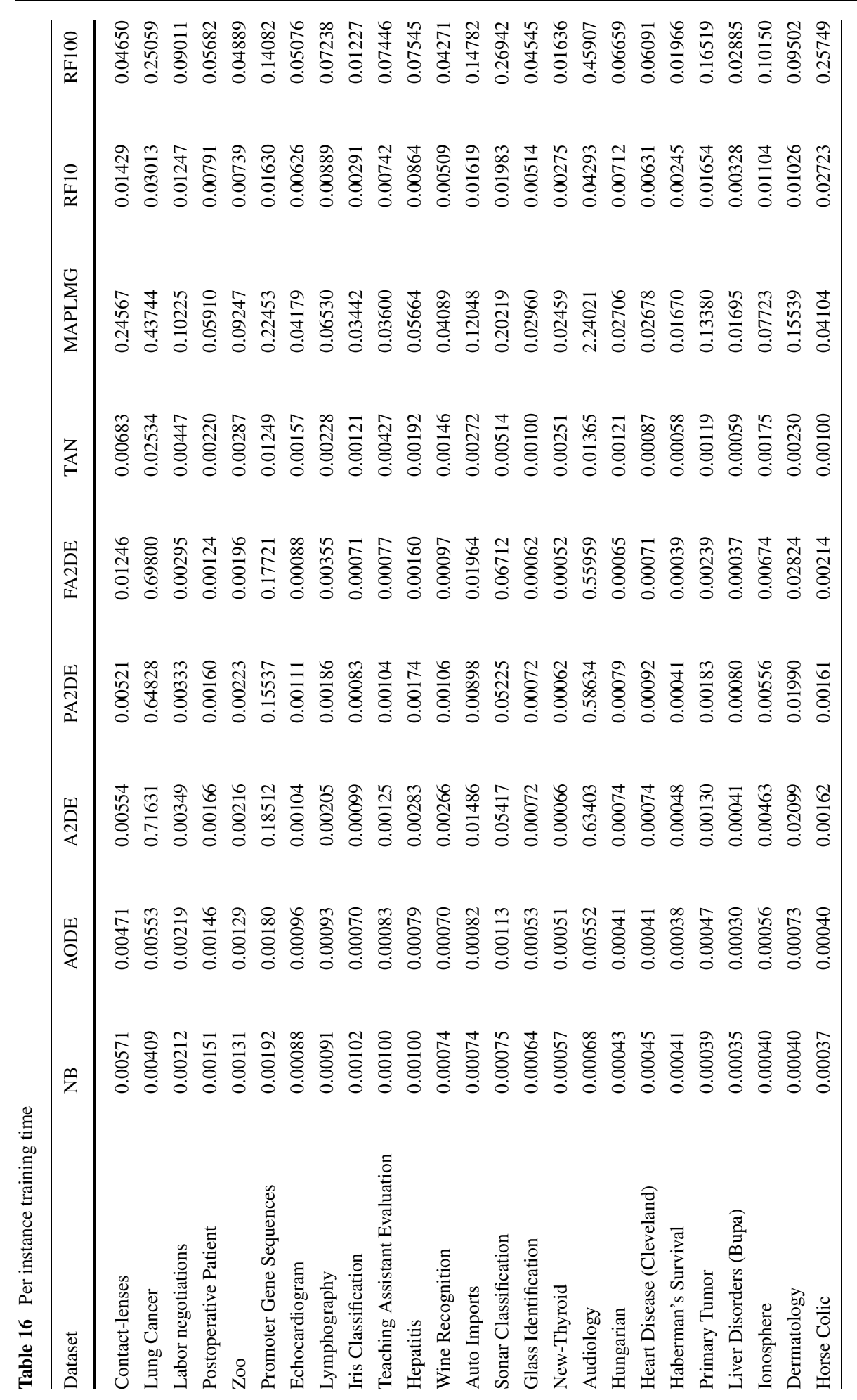




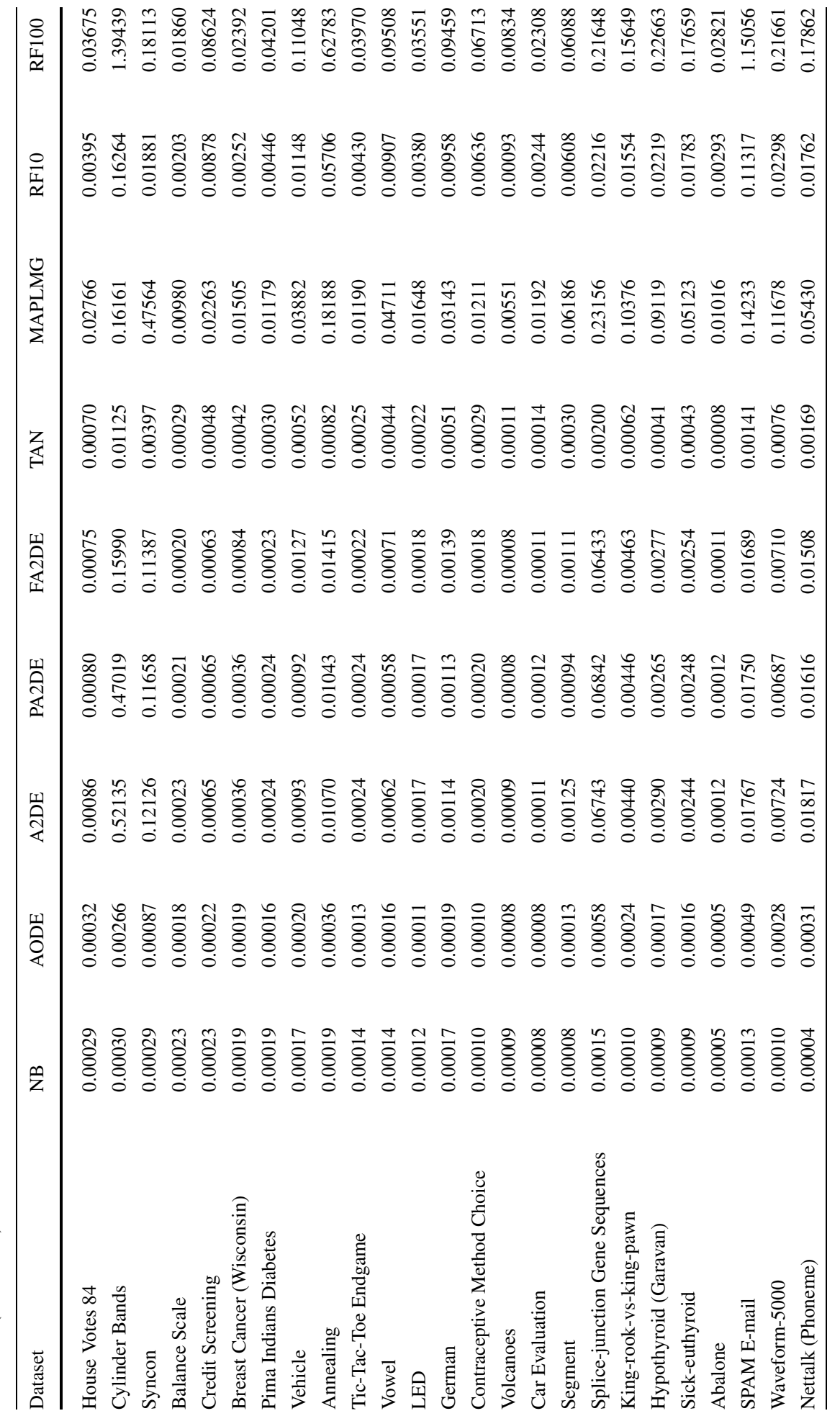




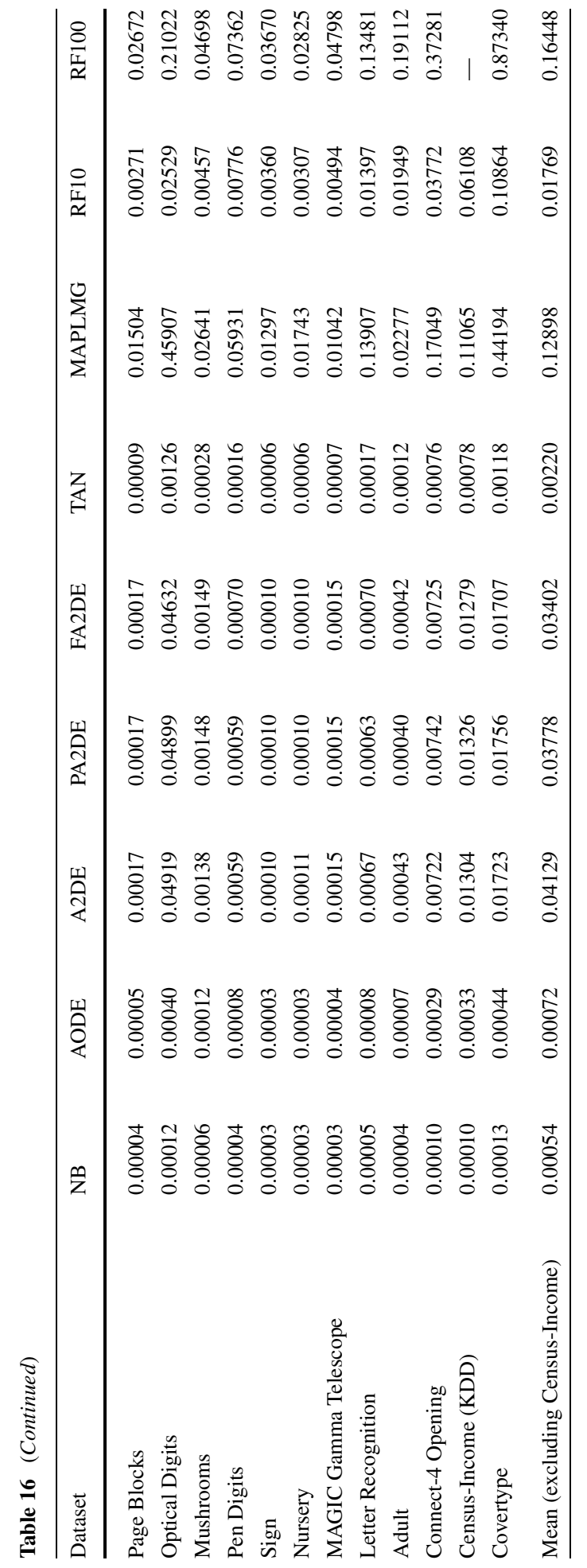




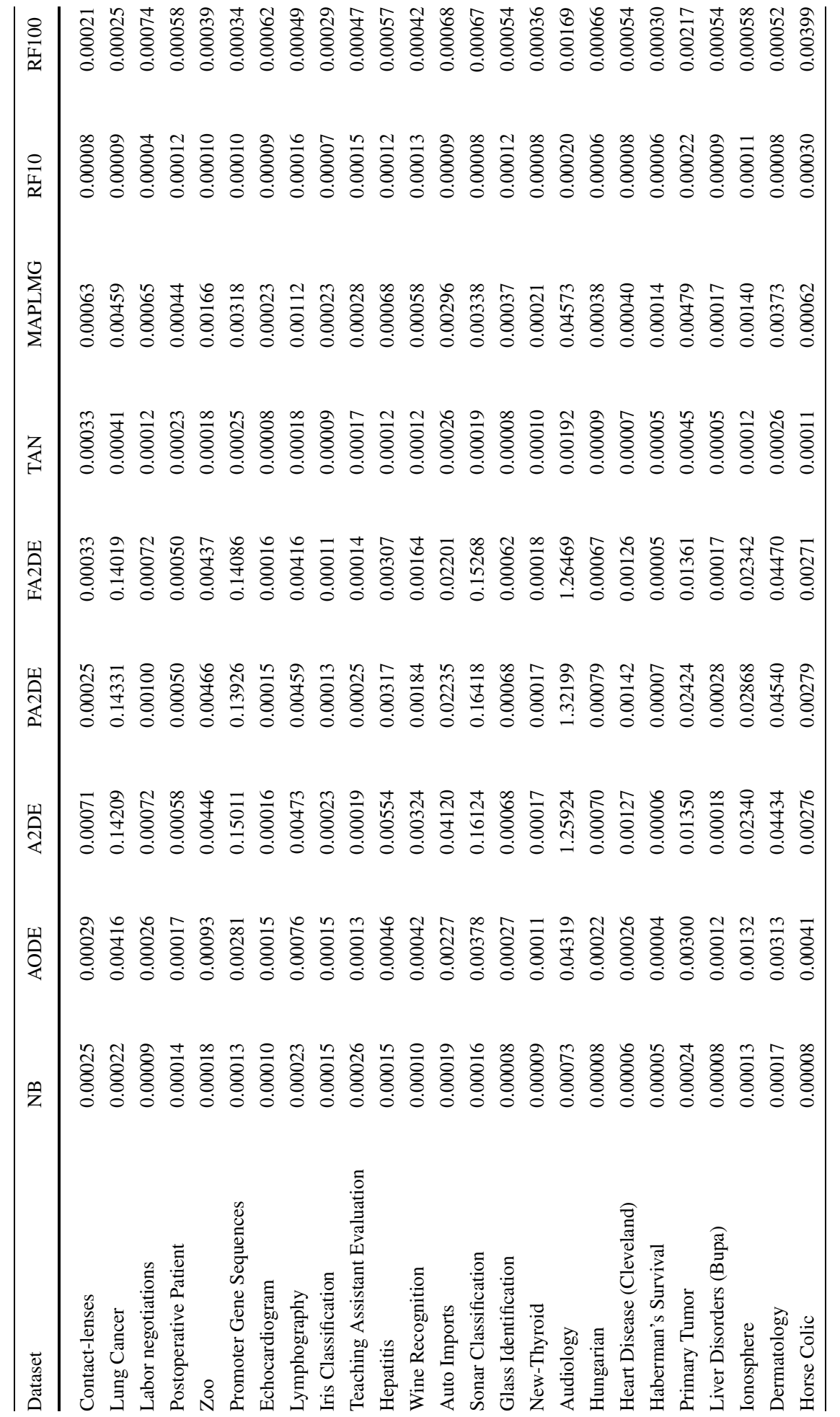




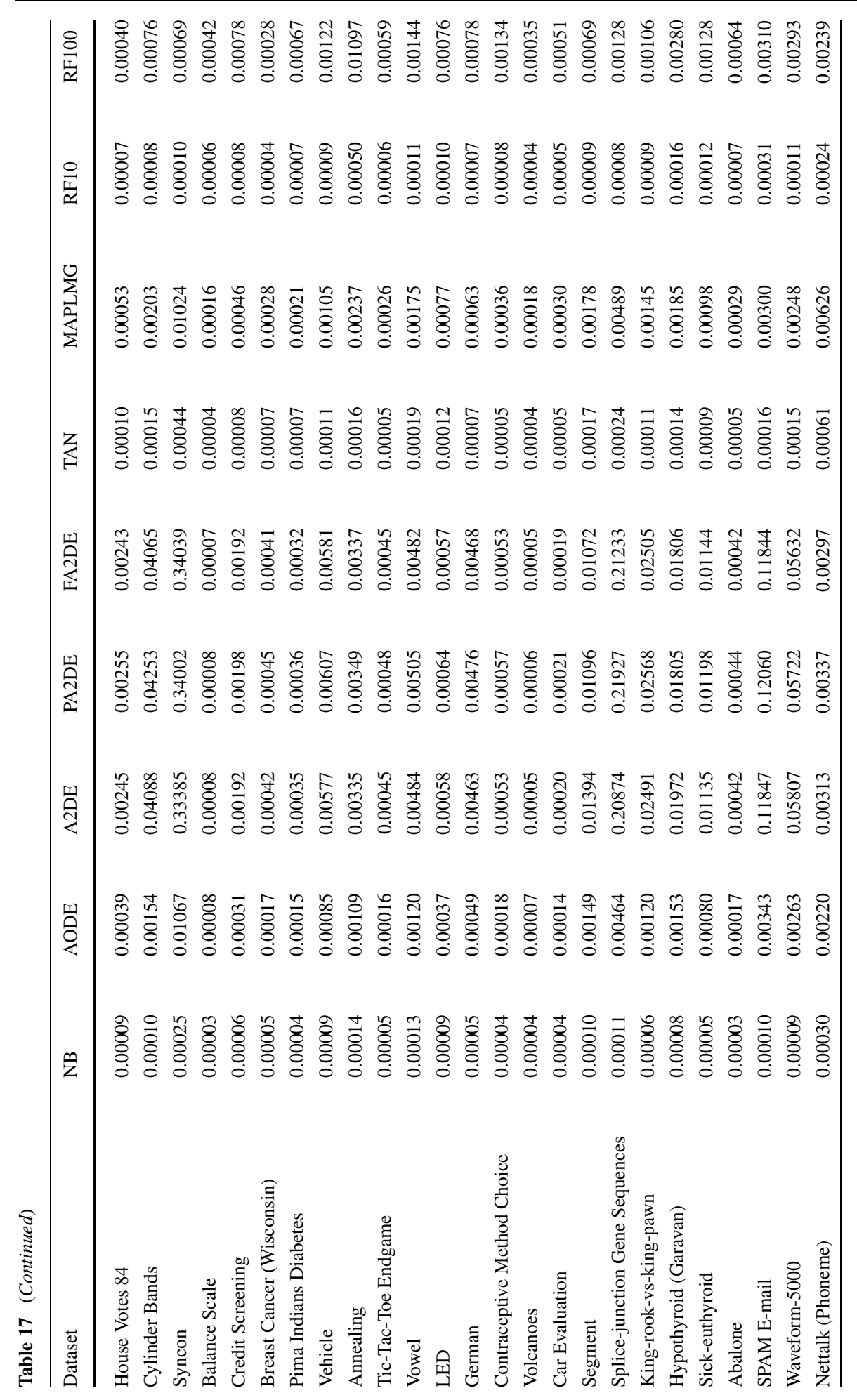




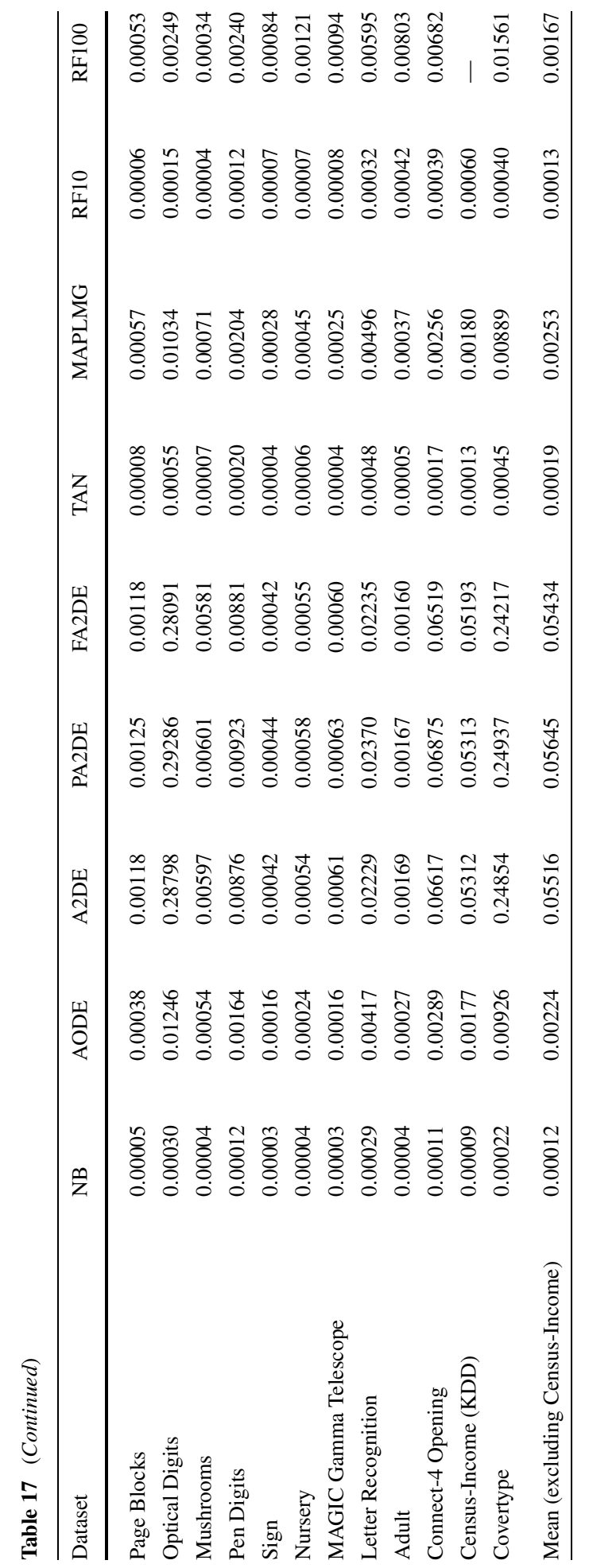




\section{References}

Affendey, L., Paris, I., Mustapha, N., Sulaiman, M., \& Muda, Z. (2010). Ranking of influencing factors in predicting students' academic performance. Information Technology Journal, 9(4), 832-837.

Birzele, F., \& Kramer, S. (2006). A new representation for protein secondary structure prediction based on frequent patterns. Bioinformatics, 22(21), 2628-2634.

Brain, D., \& Webb, G. I. (2002). The need for low bias algorithms in classification learning from large data sets. In Proceedings of the sixth European conference on principles of data mining and knowledge discovery (PKDD) (pp. 62-73). Berlin: Springer.

Breiman, L. (2001). Random forests. Machine Learning, 45, 5-32.

Camporelli, M. (2006). Using a Bayesian classifier for probability estimation: analysis of the AMIS score for risk stratification in myocardial infarction. Diploma thesis, Department of Informatics, University of Zurich.

Cerquides, J., \& Mántaras, R. L. D. (2005). Robust Bayesian linear classifier ensembles. In Proceedings of the sixteenth European conference on machine learning (pp. 70-81).

Cestnik, B. (1990). Estimating probabilities: a crucial task in machine learning. In Proceedings of the ninth European conference on artificial intelligence (pp. 147-149). London: Pitman.

Domingos, P., \& Pazzani, M. J. (1996). Beyond independence: conditions for the optimality of the simple Bayesian classifier. In Proceedings of the thirteenth international conference on machine learning (pp. 105-112). San Mateo: Morgan Kaufmann.

Fayyad, U. M., \& Irani, K. B. (1993). Multi-interval discretization of continuous-valued attributes for classification learning. In Proceedings of the thirteenth international joint conference on artificial intelligence (pp. 1022-1029). San Mateo: Morgan Kaufmann.

Ferrari, L. D., \& Aitken, S. (2006). Mining housekeeping genes with a naive Bayes classifier. BMC Genomics, $7(1), 277$.

Flikka, K., Martens, L., Vandekerckhove, J., Gevaert, K., \& Eidhammer, I. (2006). Improving the reliability and throughput of mass spectrometry-based proteomics by spectrum quality filtering. Proteomics, 6(7), 2086-2094.

Flores, M., Gámez, J., Martínez, A., \& Puerta, J. (2009). GAODE and HAODE: two proposals based on AODE to deal with continuous variables. In Proceedings of the 26th annual international conference on machine learning (pp. 313-320). New York: ACM.

Friedman, N., Geiger, D., \& Goldszmidt, M. (1997). Bayesian network classifiers. Machine Learning, 29(2), $131-163$.

Garcia, B., Aler, R., Ledezma, A., \& Sanchis, A. (2008). Protein-protein functional association prediction using genetic programming. In Proceedings of the tenth annual conference on genetic and evolutionary computation (pp. 347-348). New York: ACM.

García-Jiménez, B., Juan, D., Ezkurdia, I., Andrés-León, E., \& Valencia, A. (2010). Inference of functional relations in predicted protein networks with a machine learning approach. PLOS ONE, 4, e9969.

Hopfgartner, F., Urruty, T., Lopez, P., Villa, R., \& Jose, J. (2010). Simulated evaluation of faceted browsing based on feature selection. Multimedia Tools and Applications, 47(3), 631-662.

Hunt, K. (2006). Evaluation of novel algorithms to optimize risk stratification scores in myocardial infarction. $\mathrm{PhD}$ thesis, Department of Informatics, University of Zurich.

Jiang, L., \& Zhang, H. (2006). Weightily averaged one-dependence estimators. In PRICAI 2006: trends in artificial intelligence (pp. 970-974).

Kunchevaa, L. I., Vilas, V. J. D. R., \& Rodríguezc, J. J. (2007). Diagnosing scrapie in sheep: a classification experiment. Computers in Biology and Medicine, 37(8), 1194-1202.

Kurz, D., Bernstein, A., Hunt, K., Radovanovic, D., Erne, P., Siudak, Z., \& Bertel, O. (2009). Simple point-ofcare risk stratification in acute coronary syndromes: the AMIS model. British Medical Journal, 95(8), 662.

Langley, P., \& Sage, S. (1994). Induction of selective Bayesian classifiers. In Proceedings of the tenth conference on uncertainty in artificial intelligence (pp. 399-406). San Mateo: Morgan Kaufmann.

Lasko, T. A., Atlas, S. J., Barry, M. J., \& Chueh, K. H. C. (2006). Automated identification of a physician's primary patients. Journal of the American Medical Informatics Association, 13(1), 74-79.

Lau, Q. P., Hsu, W., Lee, M. L., Mao, Y., \& Chen, L. (2007). Prediction of cerebral aneurysm rupture. In Proceedings of the nineteenth IEEE international conference on tools with artificial intelligence (pp. 350-357). Washington: IEEE Computer Society.

Leon, A., et al. (2009). EcID. A database for the inference of functional interactions in E. coli. Nucleic Acids Research, 37, D629 (Database issue).

Liew, C., Ma, X., \& Yap, C. (2010). Consensus model for identification of novel PI3K inhibitors in large chemical library. Journal of Computer-Aided Molecular Design, 24(2), 131-141. 
Masegosa, A., Joho, H., \& Jose, J. (2007). Evaluating query-independent object features for relevancy prediction. In Advances in information retrieval (pp. 283-294).

Mitchell, T. M. (1982). Generalization as search. Artificial Intelligence, 18(2), 203-226.

Nikora, A. P. (2005). Classifying requirements: towards a more rigorous analysis of natural-language specifications. In Proceedings of the sixteenth IEEE international symposium on software reliability engineering (pp. 291-300). Washington: IEEE Computer Society.

Orhan, Z., \& Altan, Z. (2006). Impact of feature selection for corpus-based WSD in Turkish. In Proceedings of the fifth Mexican international conference on artificial intelligence (pp. 868-878). Berlin: Springer.

Pazzani, M. J. (1996). Constructive induction of Cartesian product attributes. In ISIS: information, statistics and induction in science (pp. 66-77).

Sahami, M. (1996). Learning limited dependence Bayesian classifiers. In Proceedings of the second international conference on knowledge discovery in databases (pp. 334-338). Menlo Park: AAAI Press.

Shahri, S., \& Jamil, H. (2009). An extendable meta-learning algorithm for ontology mapping. In Flexible query answering systems (pp. 418-430).

Simpson, M., Demner-Fushman, D., Sneiderman, C., Antani, S., \& Thoma, G. (2009). Using non-lexical features to identify effective indexing terms for biomedical illustrations. In Proceedings of the 12th conference of the European chapter of the association for computational linguistics (pp. 737-744). Association for Computational Linguistics.

Tian, Y., Chen, C., \& Zhang, C. (2008). Aode for source code metrics for improved software maintainability. In Fourth international conference on semantics, knowledge and grid (pp. 330-335).

Ting, K. M., Wells, J. R., Tan, S. C., Teng, S. W., \& Webb, G. I. (2011). Feature-subspace aggregating: ensembles for stable and unstable learners. Machine Learning, 82(3), 375-397.

Wang, H., Klinginsmith, J., Dong, X., Lee, A., Guha, R., Wu, Y., Crippen, G., \& Wild, D. (2007). Chemical data mining of the NCI human tumor cell line database. Journal of Chemical Information and Modeling, 47(6), 2063-2076.

Webb, G. I. (2000). Multiboosting: a technique for combining boosting and wagging. Machine Learning, 40(2), 159-196.

Webb, G. I., Boughton, J., \& Wang, Z. (2005). Not so naive Bayes: aggregating one-dependence estimators. Machine Learning, 58(1), 5-24.

Witten, I. H., \& Frank, E. (2005). Data mining: practical machine learning tools and techniques. San Mateo: Morgan Kaufmann.

Yang, Y., Webb, G., Cerquides, J., Korb, K., Boughton, J., \& Ting, K. M. (2006). To select or to weigh: a comparative study of model selection and model weighing for SPODE ensembles. In Proceedings of the seventeenth European conference on machine learning (pp. 533-544). Berlin: Springer.

Yang, Y., Webb, G. I., Cerquides, J., Korb, K. B., Boughton, J., \& Ting, K. M. (2007). To select or to weigh: a comparative study of linear combination schemes for superparent-one-dependence estimators. IEEE Transactions on Knowledge and Data Engineering, 19(12), 1652-1665.

Yang, Y., Webb, G. I., Korb, K., \& Ting, K.-M. (2007). Classifying under computational resource constraints: anytime classification using probabilistic estimators. Machine Learning, 69(1), 35-53.

Zheng, Z., \& Webb, G. I. (2000). Lazy learning of Bayesian rules. Machine Learning, 41(1), 53-84.

Zheng, F., \& Webb, G. I. (2006). Efficient lazy elimination for averaged-one dependence estimators. In Proceedings of the twenty-third international conference on machine learning (pp. 1113-1120). New York: ACM.

Zheng, F., \& Webb, G. I. (2007). Finding the right family: parent and child selection for averaged onedependence estimators. In Proceedings of the eighteenth European conference on machine learning (pp. 490-501). Berlin: Springer. 